\title{
ALCALÓIDES INDÓLICOS ISOLADOS DE ESPÉCIES DO GÊNERO Aspidosperma (APOCYNACEAE)
}

\author{
Maria de M. Pereira \\ Departamento de Medicamentos e Alimentos, Universidade Federal do Amazonas, Rua Com. Alexandre Amorim, 330, \\ 69010-300 Manaus - AM, Brasil \\ Rose Lisieux R. P. Jácome
}

Faculdade de Farmácia, Universidade Federal de Minas Gerais, Av. Pres. Antônio Carlos, 6627, 31270-901 Belo Horizonte - MG, Brasil

Antônio Flávio de C. Alcântara, Rosemeire B. Alves* e Délio S. Raslan

Departamento de Química, Instituto de Ciências Exatas, Universidade Federal de Minas Gerais, Av. Pres. Antônio Carlos, 6627, 31270-901 Belo Horizonte - MG, Brasil

Recebido em 20/7/06; aceito em 11/9/06; publicado na web em 28/5/07

\begin{abstract}
INDOLE ALKALOIDS FROM SPECIES OF THE Aspidosperma (Apocynaceae) GENUS. Species from genus Aspidosperma (Apocynaceae) are popularly employed to treat various diseases. This genus is characterized by the occurrence of indole alkaloids. Taking into account the various biological activities attributed to these alkaloids, the description of the chemical diversity in genus Aspidosperma is important. A review of simple carbolinic alkaloids isolated from species of various genera was published in 1979. In 1987, it was published another one dealing with the relationships between the chemical structures of the indole alkaloids and the evolution of Aspidosperma species. This work updates the information about the indole alkaloids isolated from Aspidosperma species.
\end{abstract}

Keywords: Apocynaceae; Aspidosperma; indole alkaloids.

\section{INTRODUÇÃO}

Plantas da família Apocynaceae estão incluídas fitogeneticamente na ordem Gentiales e subclasse Asteridae, sendo consideradas como espécies dicotiledôneas bem evoluídas e são caracterizadas normalmente pela presença de látex ${ }^{1}$. Essa família contém entre 3700 a 5100 espécies em 250 a 550 gêneros ${ }^{2,3}$, sendo encontradas predominantemente nos trópicos e subtrópicos e são menos frequientes em regiões temperadas ${ }^{4,5}$. Na flora brasileira são catalogadas como apocináceas mais de 400 espécies em 41 gêneros, sendo 32 destes encontrados apenas na Amazônia ${ }^{6}$.

$\mathrm{Na}$ família Apocynaceae, as espécies do gênero Aspidosperma são encontradas apenas na América, principalmente entre o México e a Argentina7. Em 1951, Woodson classificou 52 espécies de Aspidosperma em 9 séries e, em 1983, outras 18 espécies foram incluídas nesse gênero, segundo Robert ${ }^{8}$. Em 1987, uma nova classificação quimiotaxonômica das espécies nesse gênero foi proposta por Bolzani ${ }^{9}$, compreendendo 48 espécies em 7 séries (Tabela 1). Nessa Tabela foi incluída também uma outra série (Tomentosa), conforme descrito por Garcia ${ }^{10}$.

Além da qualidade de suas madeiras ${ }^{5,11}$, as cascas de espécies do gênero Aspidosperma são usadas comumente na forma de infusões pela medicina popular da Região Amazônica. A baixa toxicidade e a ausência de contra-indicações dessas infusões têm contribuído enormemente para difusão desse uso das cascas de Aspidosperma ${ }^{12-14}$. Como exemplos, a espécie $A$. ramiflorum é empregada no tratamento de leishmaniose ${ }^{14}$. A espécie A. nitidum é utilizada como anticonceptiva ${ }^{5}$, no tratamento de inflamações de útero e ovário, em diabetes, em problemas estomacais, contra câncer, febre e reumatismo $^{13}$. Cascas de A. nitidum, A. album, A. discolor, A. excelsum e A. polineuron são usadas por nativos de diferentes locais da Amazônia

*e-mail: brondi@netuno.lcc.ufmg.br no tratamento da malária ${ }^{15-17}$. A atividade antimalárica atribuída a essa espécie foi comprovada por testes in vitro e in vivo ${ }^{18}$. Na Colômbia, o látex dessa espécie é utilizado pelos índios Makuna e Taiwano no tratamento da hanseníase ${ }^{5}$. Cascas de A. quebrachoblanco são usadas na região andina como afrodisíaco e contra febre ${ }^{19-21}$. No Brasil, essa espécie é usada também em casos de enfisema, bronquite e pneumonia, bem como no tratamento de impotência, contra sintomas da hiperplasia prostática benigna e em dispnéia asmática e cardíaca ${ }^{20-22}$.

A família Apocynaceae caracteriza-se quimicamente pela ocorrência freqüente de estruturas alcaloídicas. No caso de espécies de Aspidosperma, há predominantemente a ocorrência de alcalóides indólicos de considerável diversidade estrutural, muitos deles contendo esqueleto $\beta$-carbolínico simples, com sistemas tricíclicos de anéis pirido-indólicos ${ }^{23}$. A marcação isotópica de precursores indica que a parte indólica desses alcalóides é derivada biossinteticamente do triptofano e a parte não indólica, provavelmente de carboidratos, pela via chiquimato ${ }^{24}$. Segundo a literatura, alcalóides indólicos constituem bons marcadores quimiotaxonômicos para classificação botânica das espécies de Aspidosperma ${ }^{10,25-27}$.

Biologicamente, muitos alcalóides indólicos agem provavelmente nos sistemas neurotransmissores opiáceos, GABAérgicos, colinérgicos, muscarínicos, serotoninérgicos e dopaminérgicos ${ }^{28,29}$. Por isso, são empregados largamente como hipotensor arterial, simpatolítico, diurético, vasoconstrictor periférico, estimulante respiratório, anestésico, agente bloqueador adrenérgico, espasmogênico intestinal, sedativo e relaxante do músculo esquelético ${ }^{20,30,31}$. Além disso, são responsáveis pelos efeitos alucinógenos do tabaco, de bebidas e rapés utilizados por nativos da Amazônia, bem como pelas propriedades sedativas do maracujá23 .

Ensaios biológicos de muitos alcalóides monoterpênicos isolados das cascas de A. quebracho-blanco ${ }^{22}$ indicaram atividade bloqueadora $\alpha$-adrenérgica e ação inibitória de contrações de mús- 
Tabela 1. Classificação das espécies do gênero Aspidosperma,* segundo refs. 9 e 10

\begin{tabular}{ll}
\hline Série & Espécies \\
\hline Rigida & A. rigidum Rusby (= A. Laxiflorum Kuhlm.) \\
Nitida & A. auriculatum Mgf., A. carapanauba M. Pichon, A. compactinervium Kuhlmann, A. discolor A. DC., A. eburneum \\
& F. Allem, A. excelsum Benth, A. marcgravianum Woodson, A. nitidum Benth ex Müll.-Arg., A. oblongum A. DC. e \\
Quebrachines & A. spegazzinii Molf. Ex Meyer \\
Polyneura & A. chakensis Spegazzini e A. quebracho-blanco Schlecht \\
Pyricolla & A. cuspa Blake, A. cylindrocarpon Müell.-Arg., A. dispermum, A. peroba, A. polyneuron Müll.-Arg. e A. sessiliflorum \\
& A. australe Müll.-Arg., A. campus-belus A. P. Duarte, A. gomesianum A. DC., A. multiflorum A. DC., A. nigricans \\
& Handro, A. olivaceum Müll.-Arg., A. parvifolium, A. populifolium A. DC., A. pyricollum Muell.-Arg., A. pyrifolium \\
Nobile & Mart., A. quirandy, A. refractum Mart., A. rhombeosignatum, A. subincanum Mart., A. tomentosum Mart., A. ulei \\
& A. album (Vahl) R. Bent., A. desmanthum Benth ex. Müll.-Arg., A. exalatum Monachino, A. fendleri, A. limae \\
& Woodson, A. megalocarpon Müll.-Arg., A. melanocalyx Müll.-Arg., A. neblinae Monachino, A. obscurinervium \\
Macrocarpa & Azembuja, A. sandwithianum e A. spruceanum Benth \\
Tomentosa & A. duckei, A. macrocarpon Mart e A. verbascifolium Müll.-Arg. \\
\hline
\end{tabular}

* Segundo Woodson, no gênero Aspidosperma são incluídas também as espécies: A. formosanum A. P. Duarte, A. longepetiolatum Kuhlm., A. gilbertii A. P. Duarte e A. ramiflorum Muell. Arg.

culo liso de tecidos de diferentes animais ${ }^{19}$, além de ação hipotensora ${ }^{20} \mathrm{e}$ analgésica ${ }^{32}$. Alcalóides das cascas das raízes e das folhas de A. pyrifolium apresentam efeito hipotensivo forte na pressão arterial. Esse efeito foi atribuído a alcalóides indólicos presentes nos extratos dessa espécie ${ }^{33}$.

Muitos alcalóides indólicos isolados de espécies do gênero Aspidosperma apresentam efeitos mutagênicos em Salmonella typhimurium, em culturas de células mamárias e em células pulmonares, além de efeitos inibitórios sobre monoamino oxidase em tecido animal, crescimento e mitose de fibroblastos cardíacos ${ }^{25}$. A atividade desses alcalóides em células de eucariontes e procariontes tem sido atribuída à sua capacidade de intercalação quando interagem com o DNA ${ }^{25,34}$. Outros alcalóides são parasiticidas e apresentam citotoxicidade em células cancerosas ${ }^{28}$. Testes contra a doença de Chagas mostram que esses alcalóides são ativos contra epimastigotos de Tripanosoma cruzi. O aumento da atividade tripanosomicida de alcalóides indólicos é verificado quando há substituição na posição C-7 do anel indólico ${ }^{28}$.

Alcalóides indólicos isolados de A. marcgravianum mostraram atividades antimicrobiana e citotóxica ${ }^{35}$, enquanto aqueles isolados das cascas das raízes de A. excelsum Benth apresentaram atividade antimicrobiana frente a Bacillus subtilis e Staphylococcus aureus $^{36}$.
Uma revisão foi feita sobre os alcalóides carbolínicos simples conhecidos até 1979, isolados de diferentes gêneros de plantas da família Apocynaceae ${ }^{23}$. Uma outra revisão sobre essa classe de alcalóides de Aspidosperma foi realizada posteriormente para investigar a relação entre estrutura química e evolução das espécies no gênero. Essa revisão engloba os estudos feitos até $1987^{\circ}$. Como resultado, pôde ser proposta uma classificação das espécies de Aspidosperma em séries ${ }^{37}$.

No presente trabalho é descrito um novo levantamento sobre alcalóides indólicos em espécies do gênero Aspidosperma, tendo em vista sua importância química e biológica. Porém, pretendeu-se realizar uma descrição mais exaustiva da diversidade estrutural dos alcalóides indólicos encontrados em espécies desse gênero até 2006, de acordo com as classes de alcalóides propostas por Manske ${ }^{38}$.

\section{DESCRIÇÃO ESTRUTURAL DOS ALCALÓIDES INDÓLICOS ISOLADOS DE Aspidosperma}

Até 1956 eram conhecidos apenas quatro alcalóides indólicos isolados de espécies de Aspidosperma ${ }^{39}$. Atualmente, esse número ultrapassa a 100 alcalóides $^{40}$. As Tabelas 2 a 19 apresentam alcalóides indólicos isolados de plantas do gênero Aspidosperma e classificados segundo os critérios de Manske ${ }^{38}$.

Tabela 2. Alcalóides indólicos do tipo $\beta$-anilinoacrilato de metila (1), fendlispermina (2), espegazinina (3 e 4), aspidospermidina (5 e 6), pirifolidina (7 e 8), aspidospermina (9 a 19), aspidoscarpina (20 a 31) e limaspermina (32 a 40$)$

\begin{tabular}{|c|c|}
\hline Alcalóide & Plantas \\
\hline (-)-Vincadiformina (1) & A. macrocarpon $^{27} ;$ A. pyrifolium $^{27,33,41}$ \\
\hline Fendlispermina (2) & A. fendler $i^{9}$ \\
\hline Espegazinina (3) & A. chakensis $^{41}$ \\
\hline Espegazimidina (4) & A. chakensis $^{41}$ \\
\hline 1,2-Desidroaspidospermidina (5) & A. neblinae ${ }^{43} ;$ A. quebracho-blanco ${ }^{42}$ \\
\hline 1,2-Desidrodesacetilpirifolidina (6) & A. neblinae $e^{43}$ \\
\hline (+)-Pirifolidina (7) & $\begin{array}{l}\text { A. } \text { cylindrocarpon }^{9} ; \text { A. neblinae } e^{43} ; \text { A. pyrifolium } \\
\text { blanco }^{46,65-68} ; \text { A. refractum }^{64} ; \text { A. quebracho- }\end{array}$ \\
\hline$(+)$-Aspidospermina $(\mathbf{8})$ & 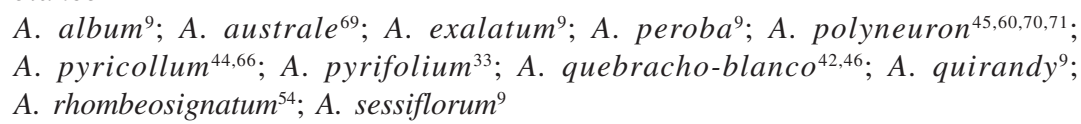 \\
\hline$(-)$-Aspidospermina $(\mathbf{9})$ & 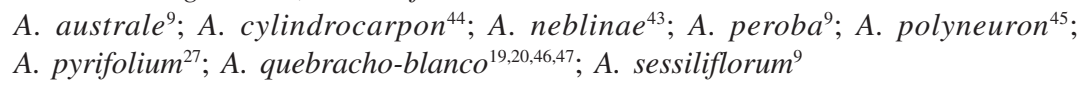 \\
\hline
\end{tabular}


Tabela 2. continuação

Alcalóide Plantas

Desacetilaspidospermina (10)

(+)-Des- $O$-metilaspidospermina (11)

$\mathrm{N}$-Metil-desacetilaspidospermina (12)

(-)-Des- $N$-metoxiaspidospermina (13)

Aspidospermidina (14)

$N$-Acetilaspidospermidina (15)

10-Metoxiaspidospermidina (16)

Desmetoxipalosina (17)

Palosina (18)

$O$-Desmetilpalosina (19)

(+)-Aspidocarpina (20)

Desacetilaspidocarpina (21)

$\mathrm{N}$-Acetil-16,17-diidroxiaspidospermidina

(Des- $O$-metilaspidocarpina, 22)

$\mathrm{N}$-propionil-16,17-diidroxi-

aspidospermidina (23)

Desacetilpirifolidina (24)

(-)-Pirifolidina (25)

Aspidolimina (26)

$\mathrm{N}$-Metilaspidospermidina (27)

$\mathrm{N}$-formilaspidospermidina (28)

Desmetoxivalesina (29)

$\mathrm{N}$-Metil-desacetildesmetoxi-

aspidospermina (30)

16-Desmetoxidesacetilpirifolidina (31)

Limaspermina (32)

16-Metoxilimaspermina (33)

15-Metoxilimaspermina (34)

Limaspermidina (35)

$N$-Acetil-limaspermina (limapodina; 36)

16-Metoxilimapodina (37)

Cilindrocarpinol (cilindrocarpol; 38)

$N$-Formilcilindrocarpinol (39)

$\mathrm{N}$-Acetilcilindrocarpinol (40)
A. neblinae $^{43}$; A. polyneuron ${ }^{9} ;$ A. pyrifolium ${ }^{27} ;$ A. quebracho-blanco $^{20,42,46,47}$

A. discolor ${ }^{48,49}$; A. eburneum ${ }^{52}$; A. excelsum ${ }^{36}$; A. neblinae ${ }^{43}$; A. pyricollum ${ }^{50}$

A. quebracho-blanco ${ }^{20}$

A. discolor ${ }^{48,49}$; A. marcgravianum ${ }^{51,53}$; A. neblinae ${ }^{43}$; A. pyrifolium $^{27}$

A. neblinae ${ }^{43} ;$ A. quebracho-blanco ${ }^{20,42}$; A. pyrifolium $^{27}$; A. rhombeosignatum ${ }^{54}$

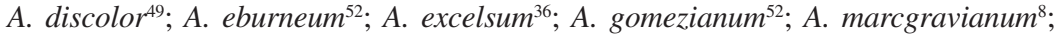

A. neblina ${ }^{9}$

A. pyrifolium $^{27}$

A. discolor ${ }^{49} ;$ A. exalatum ${ }^{9} ;$ A. limae ${ }^{49,55} ;$ A. rhombeosignatum ${ }^{54}$

A. polyneuron $^{27,45,60,61}$; A. pyrifolium ${ }^{27}$

A. exalatum ${ }^{9}$; A. limae $^{9} ;$ A. pyrifolium ${ }^{27}$

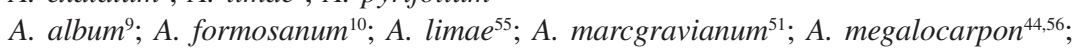

A. neblinae ${ }^{43} ;$ A. oblongum $^{8} ;$ A. obscurinervium ${ }^{57} ;$ A. $^{\text {olivaceum }}{ }^{52}$

A. megalocarpon $^{44} ; A$. obscurinervium ${ }^{57}$

A. album $^{9} ;$ A. cuspa $^{58} ;$ A. melanocalyx ${ }^{59}$

A. melanocalyx ${ }^{59}$

A. neblinae ${ }^{43} ;$ A. quebracho-blanco $o^{20,46}$

A. neblinae ${ }^{43}$; A. quebracho-blanco ${ }^{20}$

A. limae $^{55}$; A. obscurinervium ${ }^{57}$

A. quebracho-blanco ${ }^{20,46}$

A. pyrifolium $^{27}$

A. discolor ${ }^{48} ;$ A. pyrifolium $^{27}$

A. quebracho-blanco ${ }^{9}$

A. neblinae $e^{43}$

A. limae $55,62,63$

A. limae $^{55,63}$

A. album $^{9}$

A. rhombeosignatum ${ }^{54}$

A. album $^{9}$; A. limae $^{55,63}$; A. marcgravianum 8

A. limae $^{55,63}$

A. cylindrocarpon ${ }^{9}$

A. cylindrocarpon ${ }^{9}$

A. cylindrocarpon ${ }^{9}$<smiles></smiles><smiles>CC12C(Br)CCCN3CCCC1(O)C32Nc1ccccc1</smiles><smiles></smiles><smiles></smiles><smiles>[Y]c1cc([R])c2c(c1)C1(C)C(CCC3(Br)CCCN4CCC23C4)N1[Y]</smiles>

9: $\mathrm{R}^{1}=\mathrm{H} ; \mathrm{R}^{2}=\mathrm{OMe} ; \mathrm{R}^{3}=\mathrm{COMe}$ 10: $\mathrm{R}^{1}=\mathrm{R}^{3}=\mathrm{H} ; \mathrm{R}^{2}=\mathrm{OMe}$ 11: $\mathrm{R}^{1}=\mathrm{H} ; \mathrm{R}^{2}=\mathrm{OH} ; \mathrm{R}^{3}=\mathrm{COMe}$ 12: $\mathrm{R}^{1}=\mathrm{H} ; \mathrm{R}^{2}=\mathrm{OMe} ; \mathrm{R}^{3}=\mathrm{Me}$ 13: $\mathrm{R}^{1}=\mathrm{H} ; \mathrm{R}^{2}=\mathrm{H} ; \mathrm{R}^{3}=\mathrm{ROMe}$ 14: $\mathrm{R}^{1}=\mathrm{H} ; \mathrm{R}^{2}=\mathrm{OH} ; \mathrm{R}^{3}=\mathrm{H}$ 15: $\mathrm{R}^{1}=\mathrm{H} ; \mathrm{R}^{2}=\mathrm{H} ; \mathrm{R}^{3}=\mathrm{CO}_{2} \mathrm{Me}$ 16: $R^{1}=O M e ; R^{2}=R^{3}=H$ 17: $\mathrm{R}^{1}=\mathrm{R}^{2}=\mathrm{H} ; \mathrm{R}^{3}=\mathrm{COF}$ 18: $\mathrm{R}^{1}=\mathrm{H} ; \mathrm{R}^{2}=\mathrm{OMe} ; \mathrm{R}^{3}=\mathrm{COEt}$ 19: $\mathrm{R}^{1}=\mathrm{H} ; \mathrm{R}^{2}=\mathrm{OH} ; \mathrm{R}^{3}=\mathrm{COEt}$<smiles></smiles>

20: $\mathrm{R}^{1}=\mathrm{OMe} ; \mathrm{R}^{2}=\mathrm{OH} ; \mathrm{R}^{3}=\mathrm{COMe}$ 21: $R^{1}=O M e ; R^{2}=R^{3}=H$ 22: $\mathrm{R}^{1}=\mathrm{R}^{2}=\mathrm{OH} ; \mathrm{R}^{3}=\mathrm{COMe}$ 23: $\mathrm{R}^{1}=\mathrm{R}^{2}=\mathrm{OH} ; \mathrm{R}^{3}=\mathrm{COEt}$ 24: $R^{1}=R^{2}=O M e ; R^{3}=H$ 25: $\mathrm{R}^{1}=\mathrm{R}^{2}=\mathrm{OMe} ; \mathrm{R}^{3}=\mathrm{COMe}$ 26: $\mathrm{R}^{1}=\mathrm{OMe} ; \mathrm{R}^{2}=\mathrm{OH} ; \mathrm{R}^{3}=\mathrm{COEt}$ 27: $\mathrm{R}^{1}=\mathrm{H} ; \mathrm{R}^{2}=\mathrm{OH} ; \mathrm{R}^{3}=\mathrm{Me}$ 28: $\mathrm{R}^{1}=\mathrm{H} ; \mathrm{R}^{2}=\mathrm{OH} ; \mathrm{R}^{3}=\mathrm{CHO}$ 29: $\mathrm{R}^{1}=\mathrm{R}^{2}=\mathrm{H} ; \mathrm{R}^{3}=\mathrm{CHO}$ 30: $\mathrm{R}^{1}=\mathrm{R}^{2}=\mathrm{H} ; \mathrm{R}^{3}=\mathrm{Me}$ 31: $\mathrm{R}^{1}=\mathrm{OH} ; \mathrm{R}^{2}=\mathrm{OMe} ; \mathrm{R}^{3}=\mathrm{H}$<smiles></smiles><smiles>[Y]c1cc2c(c([Y])c1[R])N([Y7])C1CCC3(CCO)CCN4CCC1(C)C43C2</smiles>

32: $\mathrm{R}^{1}=\mathrm{R}^{2}=\mathrm{H} ; \mathrm{R}^{3}=\mathrm{OH} ; \mathrm{R}^{4}=\mathrm{COEt}$ 33: $\mathrm{R}^{1}=\mathrm{R}^{2}=\mathrm{OMe} ; \mathrm{R}^{3}=\mathrm{OH} ; \mathrm{R}^{4}=\mathrm{COEt}$ 34: $\mathrm{R}^{1}=\mathrm{OMe} ; \mathrm{R}^{2}=\mathrm{H} ; \mathrm{R}^{3}=\mathrm{OH} ; \mathrm{R}^{4}=\mathrm{COEt}$ 35: $\mathrm{R}^{1}=\mathrm{R}^{2}=\mathrm{H} ; \mathrm{R}^{3}=\mathrm{OH} ; \mathrm{R}^{4}=\mathrm{Me}$ 36: $\mathrm{R}^{1}=\mathrm{R}^{2}=\mathrm{H} ; \mathrm{R}^{3}=\mathrm{OH} ; \mathrm{R}^{4}=\mathrm{COMe}$ 37: $\mathrm{R}^{1}=\mathrm{H} ; \mathrm{R}^{2}=\mathrm{OMe} ; \mathrm{R}^{3}=\mathrm{OH} ; \mathrm{R}^{4}=\mathrm{COMe}$ 38: $\mathrm{R}^{1}=\mathrm{R}^{2}=\mathrm{R} ; \mathrm{R}^{3}=\mathrm{OMe} ; \mathrm{R}^{4}=\mathrm{COMe}$ 39: $R^{1}=R^{2}=H ; R^{3}=\mathrm{OMe} ; R^{4}=\mathrm{CHO}$ 40: $\mathrm{R}^{1}=\mathrm{R}^{2}=\mathrm{H} ; \mathrm{R}^{3}=\mathrm{OMe} ; \mathrm{R}^{4}=\mathrm{H}$ 
Tabela 3. Alcalóides indólicos dos tipos cilindrocarina (41 a 51) e copsano [copsanol (52 a 56), aspidosfractina (57 a 69$)$, aspidoalbina (70 a 83), acuamicina (84 e 85), condilocarpina (86 a 88), aspidospermatidina (89 a 100)]

\begin{tabular}{|c|c|}
\hline Alcalóide & Plantas \\
\hline Cilindrocarina (41) & A. cylindrocarpon ${ }^{44}$ \\
\hline 19-Hidroxicilindrocarina (42) & A. cylindrocarpon ${ }^{9}$ \\
\hline Cilindrocarpidina (43) & A. cylindrocarpon ${ }^{44,66}$ \\
\hline $\mathrm{N}$-Cinamoilcilindrocarpina & \\
\hline (cilindrocarpina; 44) & A. cylindrocarpon ${ }^{44,85}$ \\
\hline $\mathrm{N}$-Metilcilindrocarina (45) & A. cylindrocarpon ${ }^{9}$ \\
\hline $\mathrm{N}$-formilcilindrocarina $(\mathbf{4 6})$ & A. cylindrocarpon $^{9}$ \\
\hline N-benzoilcilindrocarina (47) & A. cylindrocarpon ${ }^{9}$ \\
\hline 12-Desmetoxi- $N$ - & \\
\hline acetilcilindrocarina $(\mathbf{4 8})$ & A. cylindrocarpon ${ }^{9}$ \\
\hline $\begin{array}{l}N \text {-diidrocinamoil-19-hidroxi- } \\
\text { cilindrocarina (49) }\end{array}$ & A. cylindrocarpon ${ }^{9}$ \\
\hline $\begin{array}{l}N \text {-formil-19-hidroxicilindro- } \\
\text { carina }(\mathbf{5 0})\end{array}$ & A. cylindrocarpon $^{9}$ \\
\hline $\begin{array}{l}N \text {-cinamoil-19-hidroxicilindro- } \\
\text { carina }(\mathbf{5 1})\end{array}$ & A. cylindrocarpon $^{9}$ \\
\hline 18-Epicopsanol (52) & $\begin{array}{l}\text { A. } \text { cuspa }^{58,72} ; \text { A. duckei } \\
\text { A. } \text { macrocarpon }^{27}\end{array}$ \\
\hline Copsanol (53) & $\begin{array}{l}\text { A. } \text { cuspa }^{58,72} ; \text { A. duckei }{ }^{72} \text {; } \\
\text { A. } \text { macrocarpon }^{27}\end{array}$ \\
\hline $3(S)-4(S)$ - $N$-Formilcopsanol (54) & A. verbascifolium ${ }^{9}$ \\
\hline Copsanona $(\mathbf{5 5})$ & $\begin{array}{l}\text { A. } \text { cuspa }^{58,72} ; \text { A. duckei } \\
\text { A. } \text { macrocarpon }^{27}\end{array}$ \\
\hline 10-Lactamaepicopsanol (56) & A. duckei ${ }^{9}$ \\
\hline Aspidofractinina (57) & A. refractum ${ }^{9}$ \\
\hline Aspidofractina (58) & $\begin{array}{l}\text { A. populifolium } \\
\text { A. } \\
\text { A. } \text { pyrifolium }^{65,67} \\
\text { A. } \text { refractum }^{73}\end{array}$ \\
\hline
\end{tabular}

$N$-Formil-17-metoxiaspidofractinina (59)

16,17-Dimetoxiaspidofractinina (60) (+)-Pirifolina (61)

6-Desmetoxipirifolina (62)

Refractina (valosima; 63)

Refractidina (64)

(-)-Aspidofilina (65)

17-Metoxiaspidofractinina

( $O$-metil-desacetilaspidofilina; 66)

Copsinina (67)

$\mathrm{N}$-Formil-16,17-dimetoxi-aspidofractinina $(\mathbf{6 8})$

17-Metoxiaspidofractina (69) Aspidoalbina (70)
A. populifolium ${ }^{9,52}$
A. populifolium $^{52}$
A. cylindrocarpon ${ }^{62}$;
A. pyrifolium ${ }^{33,65-67}$
A. pyrifolium ${ }^{33,44,66}$
A. populifolium $^{52}$;
A. refractum ${ }^{66,73}$
A. pyrifolium $^{67}$;
A. refractum ${ }^{67}$
A. pyrifolium $^{33,65,74}$
A. populifolium $^{52}$
A. macrocarpon $^{27}$;
A. multiflorum $^{52}$;
A. populifolium $^{52}$

\begin{tabular}{l} 
Alcalóide \\
\hline $\mathrm{N}$-Acetil- $N$-despropionilaspidoa \\
$(\mathrm{N}$-acetilaspidoalbina; 72$)$ \\
Aspidolimidina (73) \\
Des- $O$-metilaspidolimidina (74)
\end{tabular}
A. populifolium ${ }^{52}$
A. populifolium ${ }^{9}$
A. album $^{64}$;
A. desmanthum ${ }^{10}$;
A. megalocarpon $^{75,76}$;
A. spruceanum $^{52}$

$O$-Metilaspidoalbina (71)
(+)-Haplocidina (75)

Desidroxiaplocidina (76)

Haplocina (77)

Fendleridina (78)

Fendlerina (79)

21-Oxoaspidoalbina (80)

15,16-Dimetoxi-17-hidroxi-

21-oxoaspidoalbina (81)

15,16,17-Trimetoxi-

21-oxoaspidoalbina (82)

18-Oxoaplocidina (83)

Acuamicina (84)

Compactinervina (85)

Plantas

A. album $^{64}$;

A. desmanthum ${ }^{10}$;

A. spruceanum $^{52}$

A. album $^{9}$; A. limae $^{55}$;

A. marcgravianum $^{8}$;

A. megalocarpon ${ }^{75,76}$

A. album $^{9}$;

A. desmanthum ${ }^{9}$;

A. quebracho-blanco ${ }^{9}$;

A. spruceanum ${ }^{9}$

A. discolor ${ }^{48}$;

A. marcgravianum $^{8}$

A. pyrifolium $^{27}$

A. pyrifolium $^{27}$

A. flendleri ${ }^{9}$

A. album $^{9}$; A. flendleri ${ }^{9}$;

A. megalocarpon ${ }^{75,76}$;

A. quebracho-blanco ${ }^{9}$;

A. rhombeosignatum ${ }^{9}$

A. album $^{9}$; A. exalatum 77

A. exalatum $^{78}$

A. exalatum $^{78}$;

A. rhombeosignatum ${ }^{54}$

A. marcgravianum $^{8}$

A pyrifolium ${ }^{27}$;

A. quebracho-blanco ${ }^{47}$

A. compactinervium $^{52}$;

A. excelsum ${ }^{36}$;

A. pruinosum $^{25}$

Tubotaiwina (diidrocondilocarpina, 86) A. album ${ }^{9}$ A. excelsum ${ }^{36}$;

A. limae $^{55} ;$ A. pyrifolium ${ }^{27}$

11-Metoxitubotaiwina (87)

A. excelsum ${ }^{36}$

11-Metoxi-14,19-desidro-

A. populifolium ${ }^{52}$;

A. quebracho-blanco ${ }^{9}$

A. quebracho-blanco ${ }^{20}$

Aspidospermatidina (89)

$N$-Metilaspidospermatidina (90)

Desmetoxiaspidospermatina

(Acetilaspidospermatidina; 91)

$\mathrm{N}$-Acetil-12-hidroxiaspidosper-

matidina (limatinina; 92)

A. quebracho-blanco ${ }^{20,47}$

A. quebracho-blanco ${ }^{47}$

A. $\operatorname{limae}^{79,80}$;

A. subincanum ${ }^{81}$;

A. tomentosum $^{51}$

A. compactinervium $^{52}$

matidina (93)

Aspidospermatina (94)

A. oblongum ${ }^{21}$;

A. quebracho-

blanco $^{20,42,46,47}$

Desacetilaspidospermatina (95)

(+)-Limatina (96)

A. quebracho-blanco ${ }^{20,47}$

A. limae $55,79,80$;

A. marcgravianum $^{55}$

(+)-11-Metoxilimatina (97)

A. limae $^{79,80}$

(+)-11-Metoxilimatinina (98)

A. limae $^{79,80}$

14,19-Diidroaspidospermatina (99)

A. quebracho-blanco ${ }^{20}$

14,19-diidroaspidosperatina (100)

A. quebracho-blanco ${ }^{47}$ 


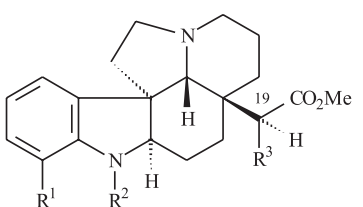

41: $\mathrm{R}^{1}=\mathrm{OMe} ; \mathrm{R}^{2}=\mathrm{R}^{3}=\mathrm{H}$

42: $\mathrm{R}^{1}=\mathrm{OMe} ; \mathrm{R}^{2}=\mathrm{H} ; \mathrm{R}^{3}=\mathrm{OH}$

43: $\mathrm{R}^{1}=\mathrm{OMe} ; \mathrm{R}^{2}=\mathrm{COMe} ; \mathrm{R}^{3}=\mathrm{H}$

44: $\mathrm{R}^{1}=\mathrm{OMe} ; \mathrm{R}^{2}=\mathrm{COCH}=\mathrm{CHC}_{6} \mathrm{H}_{5} ; \mathrm{R}^{3}=\mathrm{H}$

45: $\mathrm{R}^{1}=\mathrm{OMe} ; \mathrm{R}^{2}=\mathrm{Me} ; \mathrm{R}^{3}=\mathrm{OH}$

46: $\mathrm{R}^{1}=\mathrm{OMe} ; \mathrm{R}^{2}=\mathrm{CHO} ; \mathrm{R}^{3}=\mathrm{H}$

47: $\mathrm{R}^{1}=\mathrm{OMe} ; \mathrm{R}^{2}=\mathrm{Bz} ; \mathrm{R}^{3}=\mathrm{OH}$

48: $\mathrm{R}^{1}=\mathrm{H} ; \mathrm{R}^{2}=\mathrm{COMe} ; \mathrm{R}^{3}=\mathrm{OH}$

49: $\mathrm{R}^{1}=\mathrm{OMe} ; \mathrm{R}^{2}=\mathrm{COCH}_{2} \mathrm{CH}_{2} \mathrm{C}_{6} \mathrm{H}_{5} ; \mathrm{R}^{3}=\mathrm{H}$

50: $\mathrm{R}^{1}=\mathrm{OMe} ; \mathrm{R}^{2}=\mathrm{CHO} ; \mathrm{R}^{3}=\mathrm{OH}$

51: $\mathrm{R}^{1}=\mathrm{OMe} ; \mathrm{R}^{2}=\mathrm{COCH}=\mathrm{CHC}_{6} \mathrm{H}_{5} ; \mathrm{R}^{3}=\mathrm{OH}$

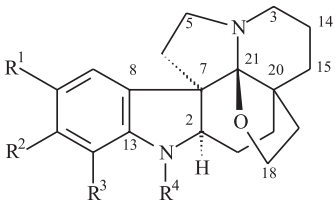

70: $\mathrm{R}^{1}=\mathrm{R}^{2}=\mathrm{OMe} ; \mathrm{R}^{3}=\mathrm{OH} ; \mathrm{R}^{4}=\mathrm{COEt}$

71: $\mathrm{R}^{1}=\mathrm{R}^{2}=\mathrm{R}^{3}=\mathrm{OMe} ; \mathrm{R}^{4}=\mathrm{COEt}$

72: $\mathrm{R}^{1}=\mathrm{R}^{2}=\mathrm{OMe} ; \mathrm{R}^{3}=\mathrm{OH} ; \mathrm{R}^{4}=\mathrm{COMe}$

73: $\mathrm{R}^{1}=\mathrm{H} ; \mathrm{R}^{2}=\mathrm{OMe} ; \mathrm{R}^{3}=\mathrm{OH} ; \mathrm{R}^{4}=\mathrm{COMe}$

74: $\mathrm{R}^{1}=\mathrm{H} ; \mathrm{R}^{2}=\mathrm{R}^{3}=\mathrm{OH} ; \mathrm{R}^{4}=\mathrm{COMe}$

75: $\mathrm{R}^{1}=\mathrm{R}^{2}=\mathrm{H} ; \mathrm{R}^{3}=\mathrm{OH} ; \mathrm{R}^{4}=\mathrm{COMe}$

76: $\mathrm{R}^{1}=\mathrm{R}^{2}=\mathrm{R}^{3}=\mathrm{H} ; \mathrm{R}^{4}=\mathrm{COMe}$

77: $\mathrm{R}^{1}=\mathrm{R}^{2}=\mathrm{H} ; \mathrm{R}^{3}=\mathrm{OH} ; \mathrm{R}^{4}=\mathrm{COE}$

78: $\mathrm{R}^{1}=\mathrm{R}^{3}=\mathrm{R}^{4}=\mathrm{H} ; \mathrm{R}^{2}=\mathrm{OMe}$

79: $\mathrm{R}^{1}=\mathrm{H} ; \mathrm{R}^{2}=\mathrm{OMe} ; \mathrm{R}^{3}=\mathrm{OH} ; \mathrm{R}^{4}=\mathrm{COEt}$

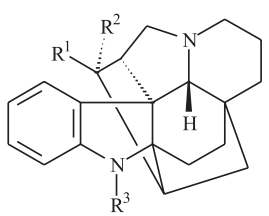

52: $\mathrm{R}^{1}=\mathrm{OH} ; \mathrm{R}^{2}=\mathrm{R}^{3}=\mathrm{H}$

53: $\mathrm{R}^{1}=\mathrm{R}^{3}=\mathrm{H} ; \mathrm{R}^{2}=\mathrm{OH}$

54: $\mathrm{R}^{1}=\mathrm{H} ; \mathrm{R}^{2}=\mathrm{OH} ; \mathrm{R}^{3}=\mathrm{CHO}$

55: $\mathrm{R}^{1}, \mathrm{R}^{2}=\mathrm{O} ; \mathrm{R}^{3}=\mathrm{H}$

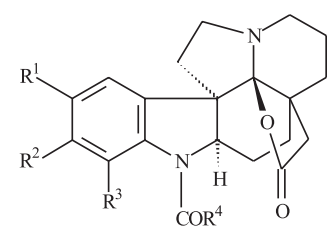

80: $\mathrm{R}^{1}=\mathrm{R}^{2}=\mathrm{OMe} ; \mathrm{R}^{3}=\mathrm{OH} ; \mathrm{R}^{4}=\mathrm{Me}$ 81: $\mathrm{R}^{1}=\mathrm{R}^{2}=\mathrm{OMe} ; \mathrm{R}^{3}=\mathrm{OH} ; \mathrm{R}^{4}=\mathrm{Et}$ 82: $R^{1}=R^{2}=R^{3}=O M e ; R^{4}=E t$

83: $\mathrm{R}^{1}=\mathrm{R}^{3}=\mathrm{H} ; \mathrm{R}^{2}=\mathrm{OH} ; \mathrm{R}^{4}=\mathrm{Ft}$<smiles>CC(=O)C1=C2Nc3ccccc3[C@]23CCN2C[C@](O)(C(C)O)[C@H]1C[C@H]23</smiles>

85<smiles>CC[C@H]1[C@@H]2CCC[C@@H]1[C@@H]1C(=C2C(C)=O)Nc2cc(OC)ccc21</smiles>

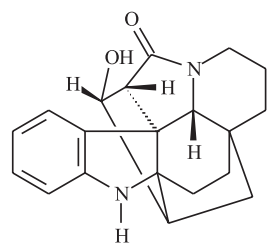

56<smiles>C/C=C1/CN2CC[C@@]34C[C@H]3C4=C1C(=O)Nc1ccccc12</smiles>

84

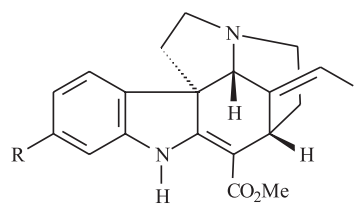
87: $R=H$
88: $R=O M e$

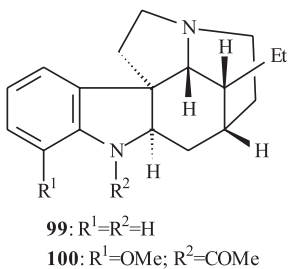

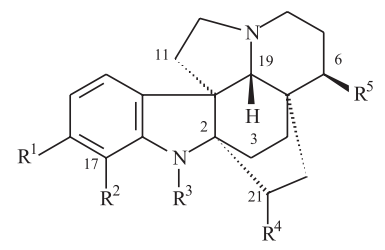

57: $\mathrm{R}^{1}=\mathrm{R}^{2}=\mathrm{R}^{3}=\mathrm{R}^{4}=\mathrm{R}^{5}=\mathrm{H}$ 58: $\mathrm{R}^{1}=\mathrm{R}^{2}=\mathrm{R}^{5}=\mathrm{H} ; \mathrm{R}^{3}=\mathrm{CHO} ; \mathrm{R}^{4}=\mathrm{CO}_{2} \mathrm{Me}$ 59: $\mathrm{R}^{1}=\mathrm{R}^{4}=\mathrm{R}^{5}=\mathrm{H} ; \mathrm{R}^{2}=\mathrm{OMe} ; \mathrm{R}^{3}=\mathrm{CHO}$ 60: $\mathrm{R}^{1}=\mathrm{R}^{2}=\mathrm{OMe} ; \mathrm{R}^{3}=\mathrm{R}^{4}=\mathrm{R}^{5}=\mathrm{H}$ 61: $\mathrm{R}^{1}=\mathrm{R}^{4}=\mathrm{H} ; \mathrm{R}^{2}=\mathrm{R}^{5}=\mathrm{OMe} ; \mathrm{R}^{3}=\mathrm{COMe}$ 62: $\mathrm{R}^{1}=\mathrm{R}^{4}=\mathrm{R}^{5}=\mathrm{H} ; \mathrm{R}^{2}=\mathrm{OMe} ; \mathrm{R}^{3}=\mathrm{COM}$ 63: $\mathrm{R}^{1}=\mathrm{R}^{5}=\mathrm{H} ; \mathrm{R}^{2}=\mathrm{OMe} ; \mathrm{R}^{3}=\mathrm{CHO} ; \mathrm{R}^{4}=\mathrm{CO}_{2} \mathrm{Me}$ 64: $\mathrm{R}^{1}=\mathrm{R}^{4}=\mathrm{R}^{5}=\mathrm{H} ; \mathrm{R}^{2}=\mathrm{OH} ; \mathrm{R}^{3}=\mathrm{COMe}$ 65: $\mathrm{R}^{1}=\mathrm{R}^{4}=\mathrm{R}^{5}=\mathrm{H} ; \mathrm{R}^{2}=\mathrm{OH} ; \mathrm{R}^{3}=\mathrm{CO}_{2} \mathrm{Me}$ 66: $\mathrm{R}^{1}=\mathrm{R}^{3}=\mathrm{R}^{5}=\mathrm{H} ; \mathrm{R}^{2}=\mathrm{OMe} ; \mathrm{R}^{4}=\mathrm{H}$ 67: $\mathrm{R}^{1}=\mathrm{R}^{2}=\mathrm{R}^{3}=\mathrm{R}^{5}=\mathrm{H} ; \mathrm{R}^{4}=\mathrm{CO}_{2} \mathrm{Me}$ 68: $\mathrm{R}^{1}=\mathrm{R}^{2}=\mathrm{OMe} ; \mathrm{R}^{3}=\mathrm{CHO} ; \mathrm{R}^{4}=\mathrm{R}^{5}=\mathrm{H}$ 69: $\mathrm{R}^{1}=\mathrm{R}^{5}=\mathrm{H} ; \mathrm{R}^{2}=\mathrm{OMe} ; \mathrm{R}^{3}=\mathrm{CHO} ; \mathrm{R}^{4}=\mathrm{CO}_{2} \mathrm{Me}$

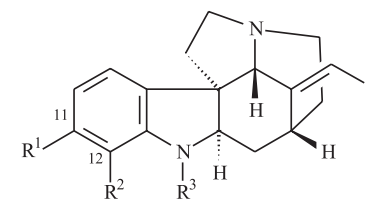

89: $\mathrm{R}^{1}=\mathrm{R}^{2}=\mathrm{R}^{3}=\mathrm{H}$

90: $R^{1}=R^{2}=H ; R^{3}=M e$

91: $\mathrm{R}^{1}=\mathrm{R}^{2}=\mathrm{H} ; \mathrm{R}^{3}=\mathrm{CO}_{2} \mathrm{Me}$

92: $\mathrm{R}^{1}=\mathrm{H} ; \mathrm{R}^{2}=\mathrm{OH} ; \mathrm{R}^{3}=\mathrm{COMe}$

93: $\mathrm{R}^{1}=\mathrm{OH} ; \mathrm{R}^{2}=\mathrm{H} ; \mathrm{R}^{3}=\mathrm{COMe}$

94: $\mathrm{R}^{1}=\mathrm{H} ; \mathrm{R}^{2}=\mathrm{OMe} ; \mathrm{R}^{3}=\mathrm{COMe}$

95: $\mathrm{R}^{1}=\mathrm{R}^{3}=\mathrm{H} ; \mathrm{R}^{2}=\mathrm{OMe}$

96: $\mathrm{R}^{1}=\mathrm{H} ; \mathrm{R}^{2}=\mathrm{OH} ; \mathrm{R}^{3}=\mathrm{COEt}$

97: $\mathrm{R}^{1}=\mathrm{OMe} ; \mathrm{R}^{2}=\mathrm{OH} ; \mathrm{R}^{3}=\mathrm{COEt}$

98: $\mathrm{R}^{1}=\mathrm{R}^{3}=\mathrm{OMe} ; \mathrm{R}^{2}=\mathrm{OH}$

Tabela 4. Alcalóides indólicos do tipo quebrachamina (101 a 103)

Alcalóide

(+)-Quebrachamina (101)

(-)-Quebrachamina (102)

$N$-Óxido de razidina (103)
Plantas

A. album $^{64}$; A. peroba 9 ;

A. polyneuron ${ }^{45,60,70,110}$;

A. pyrifolium ${ }^{33}$; A. quebrachoblanco $^{19,42,46,47}$; A. sandwithianum ${ }^{9}$

A. album $^{64}$; A. chakensis ${ }^{41}$;

A. pyrifolium $^{33}$

A. quebracho-blanco ${ }^{20,47,105}$<smiles></smiles>

101: $\mathrm{H}-5 \mathrm{~s}$ 102: H-5 5

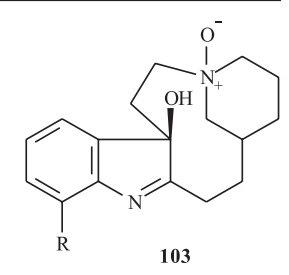

103

Tabela 5. Alcalóides indólicos do tipo elipticina-olivacina (104 a 118)

\begin{tabular}{|c|c|}
\hline Alcalóide & Plantas \\
\hline Elipticina (104) & 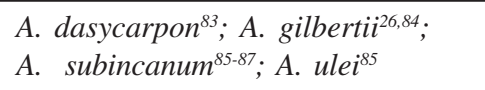 \\
\hline Olivacina (105) & 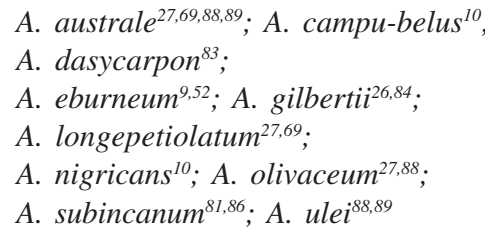 \\
\hline 9-Metoxielipticina (106) & A. dasycarpon ${ }^{83}$ \\
\hline 9-Metoxiolivacina (107) & A. vargasii ${ }^{9}$ \\
\hline$N$-Óxido de olivacina $(\mathbf{1 0 8})$ & A. nigricans $^{52}$ \\
\hline N-Metilolivacina $(\mathbf{1 0 9})$ & A. subincanum ${ }^{86}$ \\
\hline
\end{tabular}<smiles>[Y]c1ccc2[nH]c3c(C)c4ccnc([Y])c4c([Y])c3c2c1</smiles>

104: $R^{1}=M e ; R^{2}=R^{3}=H$ 105: $R^{1}=R^{3}=H ; R^{2}=M e$ 106: $\mathrm{R}^{1}=\mathrm{Me} ; \mathrm{R}^{2}=\mathrm{H} ; \mathrm{R}^{3}=\mathrm{OMe}$ 107: $\mathrm{R}^{1}=\mathrm{H} ; \mathrm{R}^{2}=\mathrm{Me} ; \mathrm{R}^{3}=\mathrm{OMe}$<smiles>[R][n+]1ccc2c(C)c3[nH]c4ccccc4c3c(C)c2c1</smiles>

A. nigricans $^{52}$

A. subincanum 
Tabela 5. continuação

\begin{tabular}{|c|c|}
\hline Alcalóide & Plantas \\
\hline$N$-Metiltetraidroelipticina $(\mathbf{1 1 0})$ & $\begin{array}{l}\text { A. dasycarpon } \\
\text { A. } \text { parvifolium }^{93,93} ; \\
\text { A. } \text { subincanum }^{81,86} ; \\
\text { A. } \text { vargasii }^{9} ; \text { A. } \text { ulei }^{85,87}\end{array}$ \\
\hline$N$-Metiltetraidroolivacina & \\
\hline [(+)-guatambuína; $\mathbf{1 1 1 ]}$ & 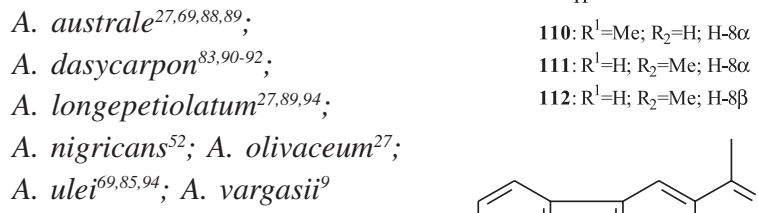 \\
\hline (-)-guatambuína (112) & A. australe 69 \\
\hline $\begin{array}{l}\text { Hidróxido de } N, N \text {-dimetiltetraidro- } \\
\text { elipticínio (113) }\end{array}$ & A. gilbertii ${ }^{26,84}$ \\
\hline 3,4-Diidroolivacina (114) & $\begin{array}{l}\text { A. dasycarpon }{ }^{90} ; \\
\text { A. subincanum } \\
86 ; \text { A. ulei }^{9}\end{array}$ \\
\hline N-metil-1,2-diidroelipticina $(\mathbf{1 1 5})$ & A. subincanum ${ }^{86}$ \\
\hline 1,2-Diidropiridocarbazol (116) & A. subincanum ${ }^{86}$ \\
\hline 1,2-Diidroelipticina $\mathbf{( 1 1 7 )}$ & $\begin{array}{l}\text { A. dasycarpon } \\
\text { A. subincanum } \\
81,86,87\end{array}$ \\
\hline 1,2-diidroolivacina (118) & A. dasycarpon ${ }^{83}$ \\
\hline
\end{tabular}

Tabela 6. Alcalóides indólicos dos tipos heteroioimbano (119 a 125) e picrafilina (126)

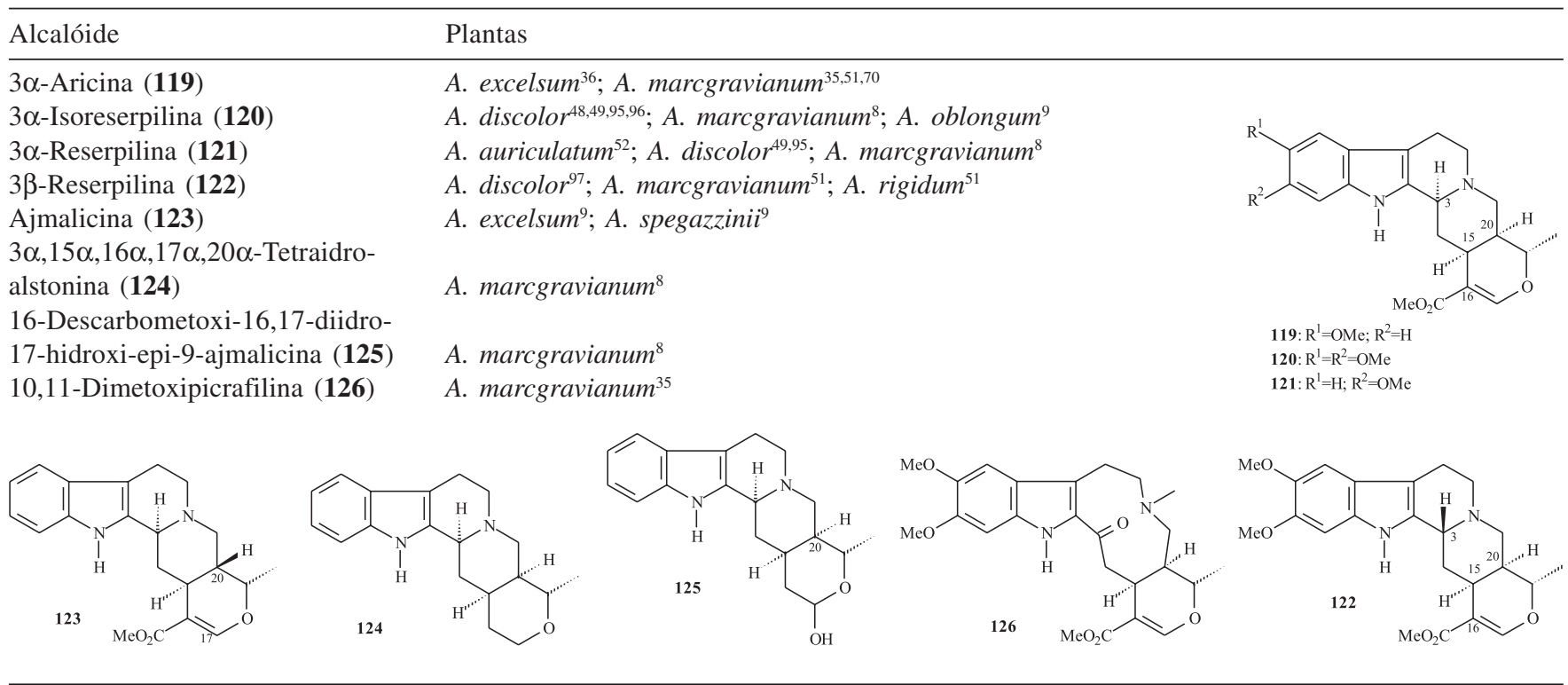

Tabela 7. Alcalóides indólicos do tipo ioimbano (127 a 145)

\begin{tabular}{|c|c|}
\hline Alcalóide & Plantas \\
\hline $3 \beta, 15 \alpha, 16 \alpha, 17 \alpha, 20 \beta$-Pseudoioimbina (127) & A. oblongum ${ }^{95}$ \\
\hline $3 \alpha, 15 \alpha, 16 \beta, 17 \alpha, 20 \beta$-Ioimbina & 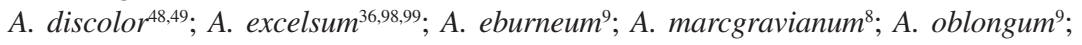 \\
\hline (ioimbina; 128) & A. peroba ${ }^{9} ;$ A. polyneuron ${ }^{60} ;$ A. pruinosum $^{25} ;$ A. $^{\text {pyricollum }}{ }^{50} ;$ A. quebracho-blanco ${ }^{42}$ \\
\hline $3 \alpha, 15 \alpha, 16 \alpha, 17 \alpha, 20 \alpha$-Aloioimbina (129) & A. marcgravianum $^{8}$ \\
\hline $3 \alpha, 15 \alpha, 16 \alpha, 17 \beta, 20 \alpha-$ & \\
\hline 17-epi-Aloioimbina (130) & A. marcgravianum ${ }^{8}$ \\
\hline $3 \alpha, 15 \alpha, 16 \beta, 17 \alpha, 20 \beta$-Ioimbina & \\
\hline$(\alpha$-ioimbina; 131) & A. marcgravianum $^{8}$ \\
\hline
\end{tabular}


Tabela 7. continuação

\begin{tabular}{|c|c|}
\hline Alcalóide & Plantas \\
\hline \multicolumn{2}{|l|}{$3 \alpha, 15 \alpha, 16 \beta, 17 \alpha, 20 \alpha$-Corinantina } \\
\hline (corinantina; 132) & A. marcgravianum $^{8}$ \\
\hline $3 \alpha, 17 \beta$-10-Metoxi- $\beta$-ioimbina (133) & A. marcgravianum ${ }^{8}$ \\
\hline \multicolumn{2}{|l|}{$3 \alpha, 15 \alpha, 16 \beta, 17 \alpha, 20 \alpha-10$-Metoxi- } \\
\hline$\alpha$-ioimbina (134) & A. marcgravianum $^{8} ;$ A. pruinosum ${ }^{25}$ \\
\hline 10-Metoxi-17-epialoioimbina (135) & A. marcgravianum $^{8}$ \\
\hline \multicolumn{2}{|l|}{$3 \alpha, 15 \alpha, 16 \beta, 17 \alpha, 20 \beta-10$-Metoxicorinantina } \\
\hline (excelsinina e aspidexcina; 136 ) & A. excelsum ex,98,99; A. marcgravianum $^{35}$ \\
\hline $3 \alpha, 15 \alpha, 16 \alpha, 17 \alpha, 20 \beta-10$-Metoxi- & \\
\hline ioimbina (137) & A. oblongum ${ }^{9} ;$ A. pruinosum ${ }^{24,25}$ \\
\hline $3 \alpha, 15 \alpha, 16 \alpha, 17 \beta, 20 \beta-Q u e b r a c h i n a$ & 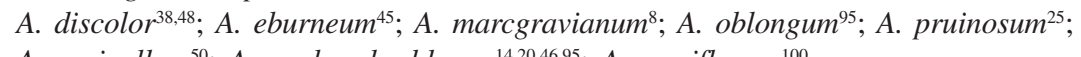 \\
\hline$[(-)-\beta$-ioimbina; 138] & A. pyricollum $^{50} ;$ A. quebracho-blanco ${ }^{14,20,46,95} ;$ A. ramiflorum $^{100}$ \\
\hline $3 \alpha, 15 \alpha, 16 \alpha, 17 \beta, 20 \alpha$-Quebrachina (139) & $\begin{array}{l}\text { A. discolor }{ }^{48} ; \text { A. eburneum }{ }^{45} ; \text { A. excelsum } \\
\text { pruinosum }\end{array}$ \\
\hline
\end{tabular}

$3 \alpha, 15 \alpha, 16 \alpha, 17 \alpha, 20 \beta-O$-Acetil-

ioimbina (140)

$N$-Óxido de $\beta$-ioimbina (141)

$3,4,5,6$-Tetradesidro- $\beta$-ioimbina (142)

A. excelsum $^{36,98,99}$; A. marcgravianum ${ }^{95}$

3,4-Desidro- $\beta$-ioimbina

(heteroioimbina; 143)

19,20-Desidro- $\beta$-ioimbina (144)

$3 \alpha, 15 \alpha, 16 \beta, 17 \alpha, 20 \alpha-19,20$-Desidro-

$\alpha$-ioimbina (145)

A. marcgravianum $^{8}$

A. marcgravianum $^{8}$

A. marcgravianum $^{8}$; A. pyricollum ${ }^{50}$

A. marcgravianum $^{8}$; A. pyricollum ${ }^{50}$

A. oblongum $^{95}$

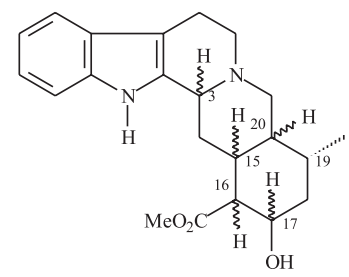

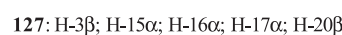

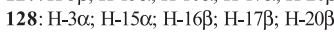
129: H-3 $\alpha ; \mathrm{H}-15 \alpha ; \mathrm{H}-16 \alpha ; \mathrm{H}-17 \alpha ; \mathrm{H}-20 \alpha$ 130: $\mathrm{H}-3 \alpha ; \mathrm{H}-15 \alpha ; \mathrm{H}-16 \alpha ; \mathrm{H}-17 \beta ; \mathrm{H}-20 \alpha$

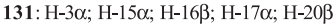
132: H-3 $\alpha ; \mathrm{H}-15 \alpha ; \mathrm{H}-16 \beta ; \mathrm{H}-17 \alpha ; \mathrm{H}-20 \alpha$

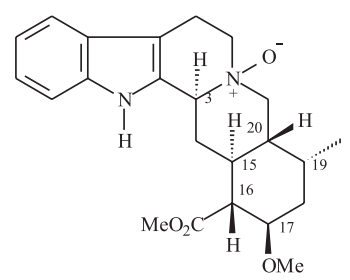

141

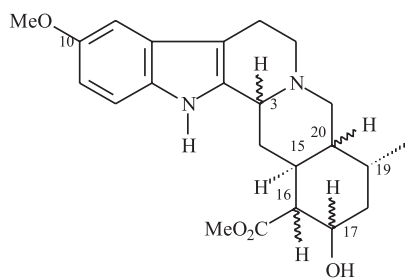

133: H-3 $\alpha$; H-16 $\alpha$; H-17 $\beta$; H-20 $\beta$ 134: H-3 $\alpha ; \mathrm{H}-16 \beta ; \mathrm{H}-17 \alpha ; \mathrm{H}-20 \alpha$

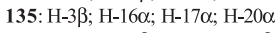
136: $\mathrm{H}-3 \alpha ; \mathrm{H}-16 \beta ; \mathrm{H}-17 \alpha ; \mathrm{H}-20 \beta$ 137: H-3 $\alpha$; H-16 $\alpha$; H-17 $\alpha$; H-20ß

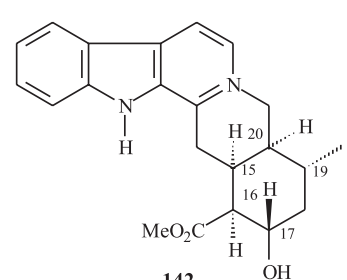

142<smiles>CC(=O)[C@H]1[C@@H](O)CC[C@H]2CN3CCc4c([nH]c5ccccc45)[C@H]3C[C@H]21</smiles>

138: $\mathrm{H}-20 \beta$ 139: $\mathrm{H}-20 \alpha$

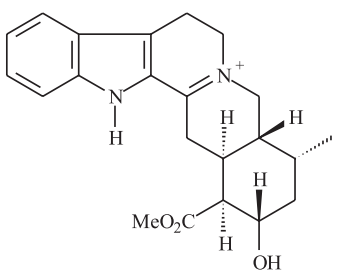

143

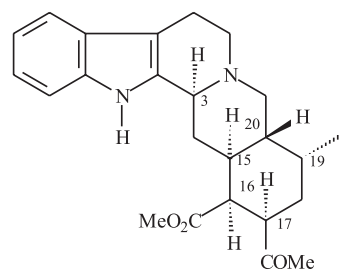

140

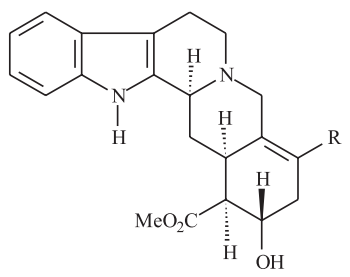

144: $\mathrm{R}=\mathrm{H}$

145: $\mathrm{R}=\mathrm{Me}$

Tabela 8. Alcalóides indólicos do tipo eburnamenina (146 a 149)

\begin{tabular}{|c|c|c|}
\hline Alcalóide & Plantas & \\
\hline Eburnamenina (146) & A. quebracho-blanco ${ }^{20,42}$ & \\
\hline$\alpha$-Hidroxieburnamonina & A. neblinae ${ }^{43} ;$ A. quebracho- & \\
\hline (eburnamina, 147) & blanco $^{20,42}$ & \\
\hline$\beta$-Hidroxieburnamonina (148) & A. quebracho-blanco ${ }^{47}$ & \\
\hline eburnamonina (149) & A. neblinae ${ }^{43} ;$ A. quebracho- & \\
\hline & blanco $^{42}$ & $\begin{array}{l}\text { 147: } R^{1}=H ; R^{2}=\mathrm{OH} \\
148: R^{1}=O H ; R^{2}=H\end{array}$ \\
\hline
\end{tabular}


Tabela 9. Alcalóides indólicos dos tipos pseudoindoxila (150 e 151), ioimbanoindol (152) e carapaubina (153)

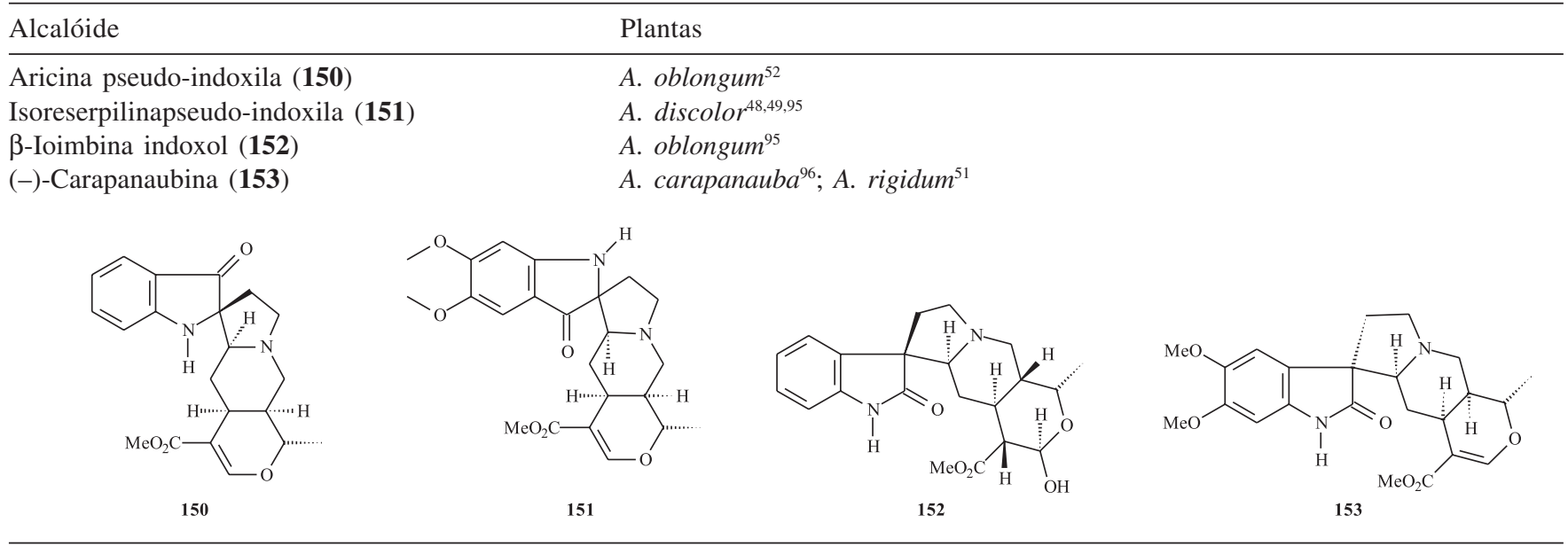

Tabela 10. Alcalóides indólicos dos tipos picralina (154 e 155) e normacusina (156 a 161$)$

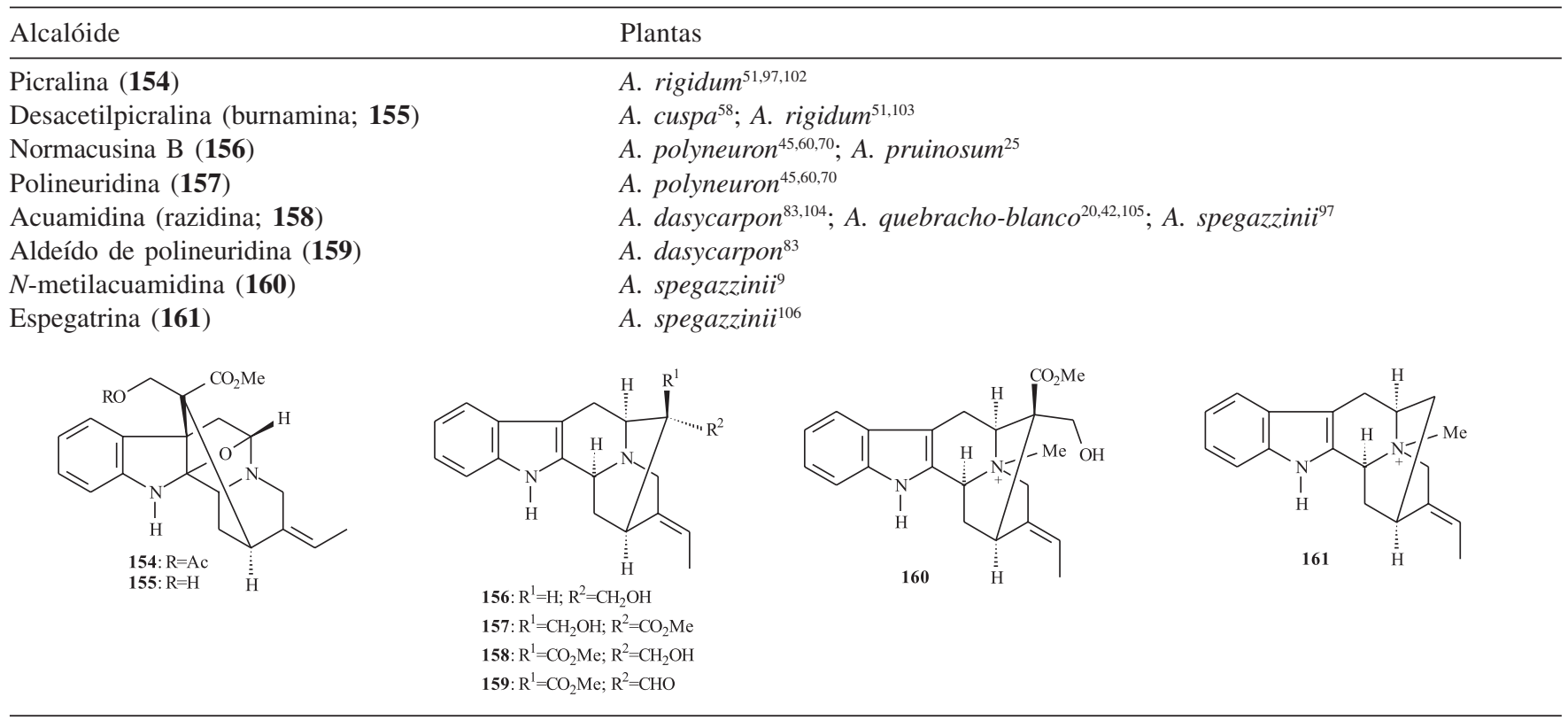

Tabela 11. Alcalóides indólicos dos tipos estemadenina (162), aspidoquibina (163) e aspidodasicarpina (164)

\begin{tabular}{ll}
\hline Alcalóide & Plantas \\
\hline (+)-Estemadenina $(\mathbf{1 6 2})$ & A. pyricollum \\
Aspidoquibina $(\mathbf{1 6 3})$ & A. quebracho-blanco ${ }^{22}$ \\
Aspidodasicarpina $(\mathbf{1 6 4})$ & A. cuspa $^{58} ;$ A. dasycarpon $^{83,90,108} ;$ A. marcgravianum $^{8}$
\end{tabular}<smiles>C=CC1CN2CCc3c([nH]c4ccccc34)C2CC1C(CO)(CO)C(C)=O</smiles>

$162 \mathrm{OH}$

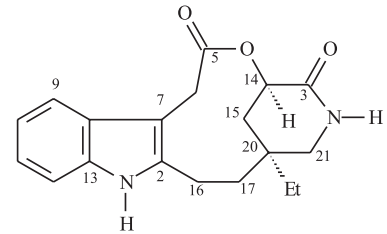

163

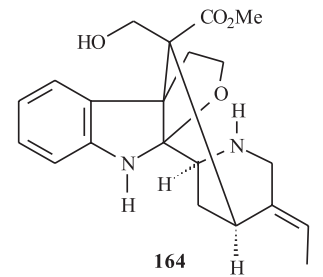


Tabela 12. Alcalóides indólicos dos tipos secodina (165 a 167) e secamina (168 a 172)

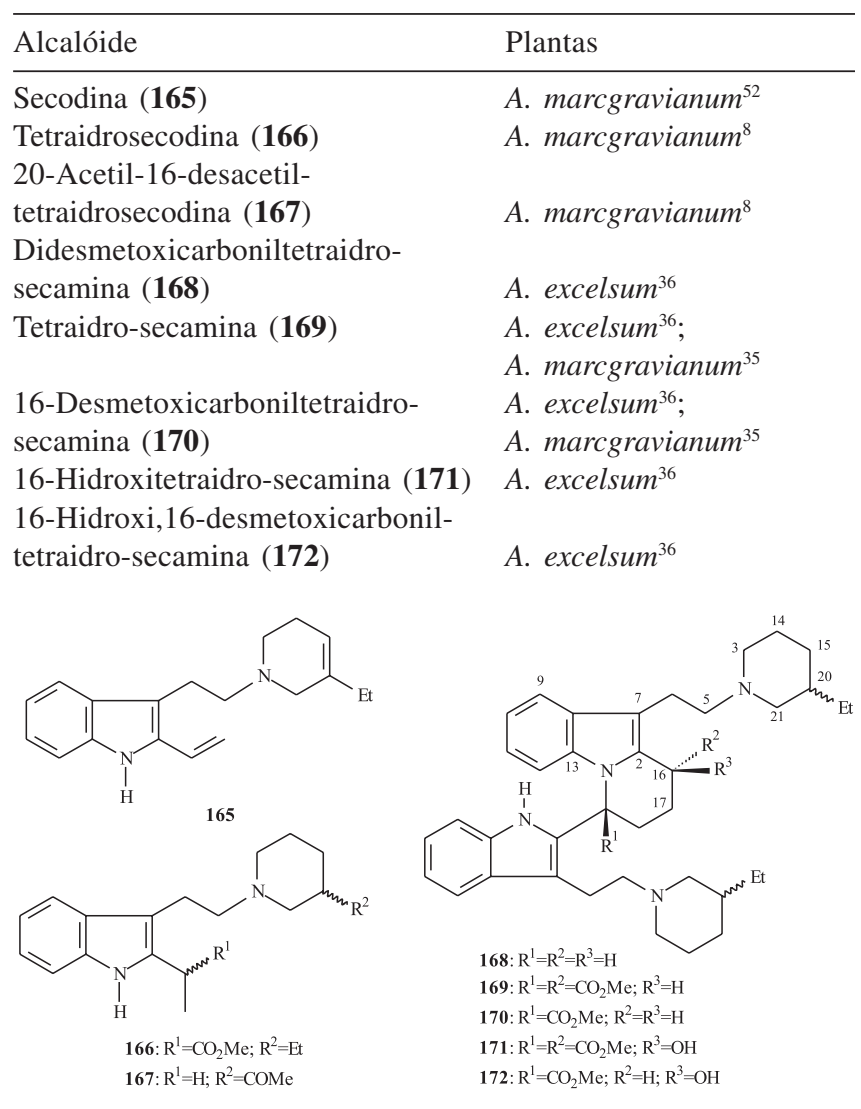

Tabela 13. Alcalóides indólicos dos tipos obscurinervina (173 e 174), obscurinervidina (175 e 176) e neblinina (177)

\begin{tabular}{ll}
\hline Alcalóide & Plantas \\
\hline Obscurinervina (173) & A. obscurinervium \\
6,7-Diidroobscurinervina (174) & A. obscurinervium \\
Obscurinervidina (175) & A. obscurinervium \\
67,109 \\
6,7-Diidroobscurinervidina (176) & A. obscurinervium \\
Neblinina (177) & A. neblinae \\
&
\end{tabular}

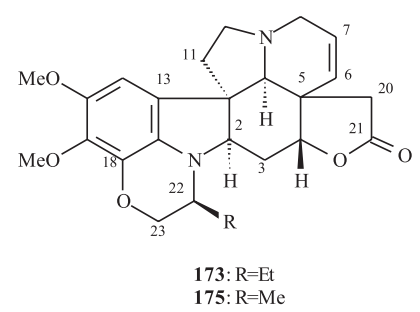

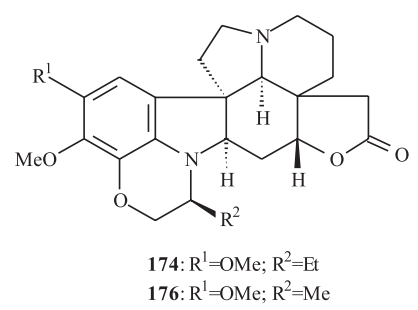<smiles>COc1ccc2c3c1OCC(C)N3CC1CC34C=CCN3CCC43CC(=O)O[C@@H]3CC21</smiles>

Tabela 14. Alcalóides indólicos do tipo harmano (178 a 186)

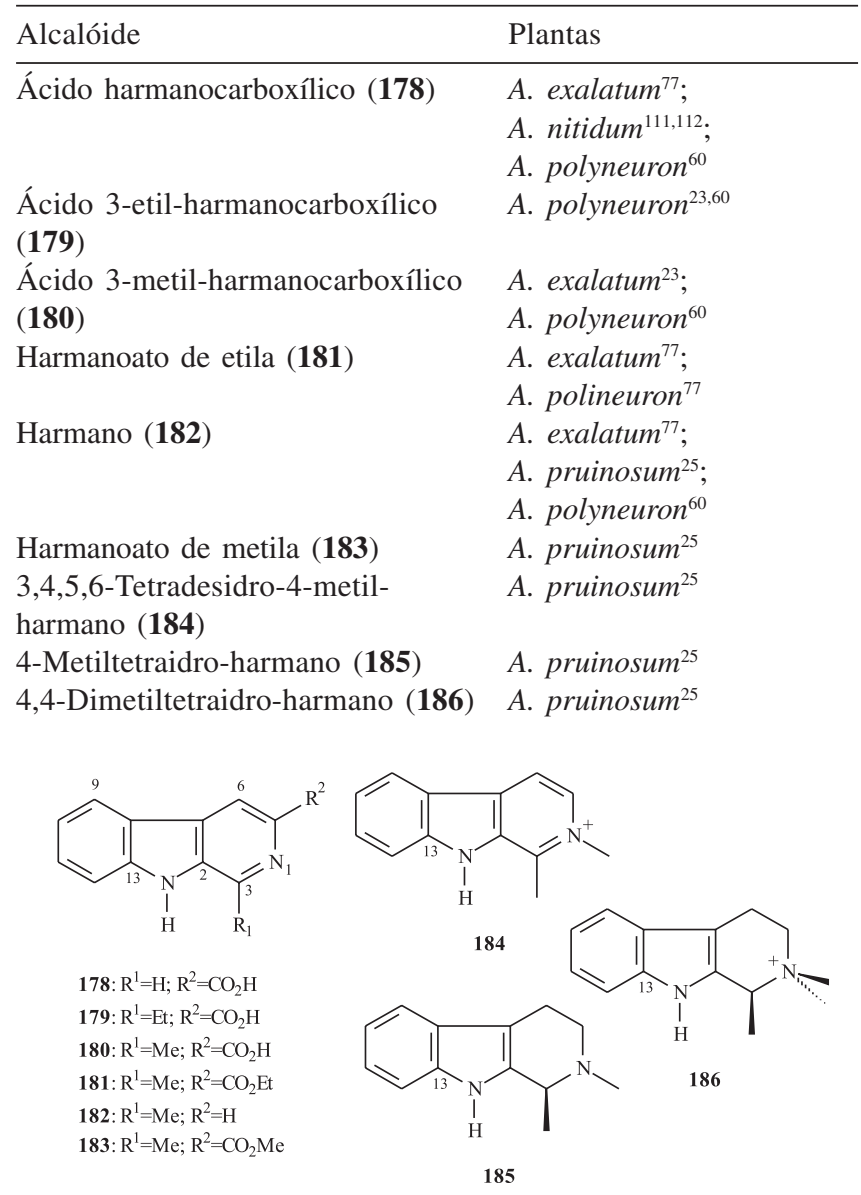

Tabela 15. Alcalóides indólicos dos tipos subincanina (187), 11Hpirido[3,4a]-carbazólio (188) e antirina (189 a 191)

\begin{tabular}{ll}
\hline Alcalóide & Plantas \\
\hline Subincanina (187) & A. subincanum $^{81}$ \\
Hidróxido de 5-etil-2-metil- & \\
11H-pirido[3,4a]-carbazólio (188) & A. gilbertii $^{26,101}$ \\
Antirina (189) & A. marcgravianum $^{8} ;$ \\
& A. oblongum $^{52}$ \\
11-Metoxiantirina (190) & A. marcgravianum \\
& A. oblongum $^{52}$ \\
derivados 18,19-diidroantirina (191) & A. marcgravianum \\
\end{tabular}<smiles>CC(=O)OC1CC2c3c(Br)cc4c([nH]c5ccccc54)c3CN(C)C2O1</smiles><smiles>C[n+]1ccc2c(Br)cc3c4ccccc4[nH]c3c2c1</smiles>

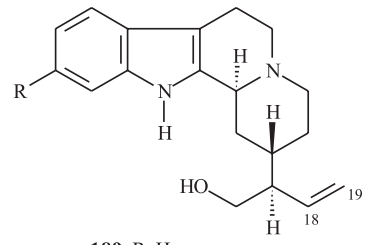<smiles>CC[C@H](CO)[C@H]1CCN2CCc3c(n([Tl])c4ccccc34)[C@@H]12</smiles> 
Tabela 16. Alcalóides indólicos dos tipos geissoschizol (192 a 196), isositsiriquina (197 a 201), sitsiriquina (202 a 208$)$, corinanteol (209 a 214), valesiachotamina (215) e deplancheína (216)

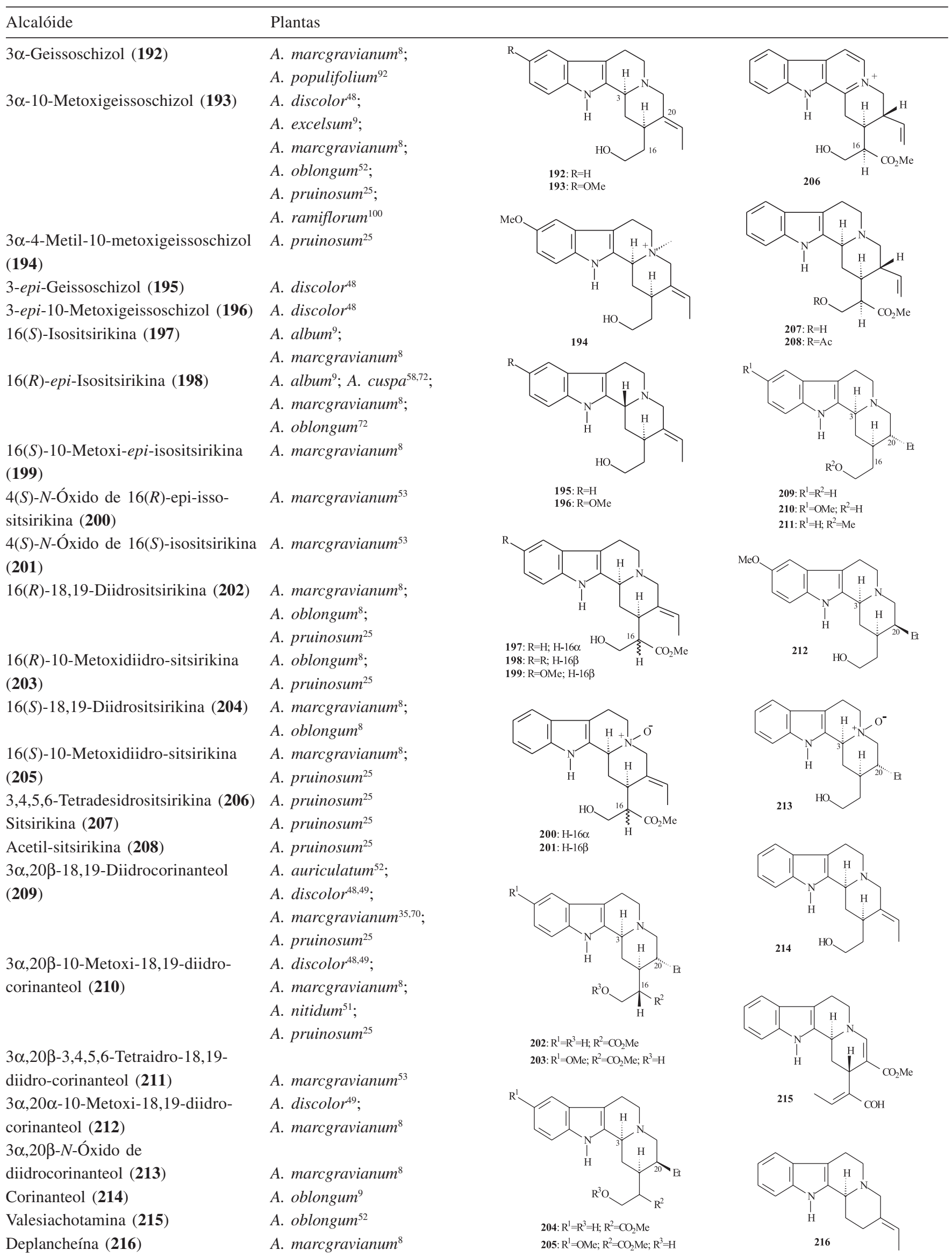


Tabela 17. Alcalóides indólicos dos tipos ocrolifuanina (217 e 218) usambarensina (219 a 228$)$

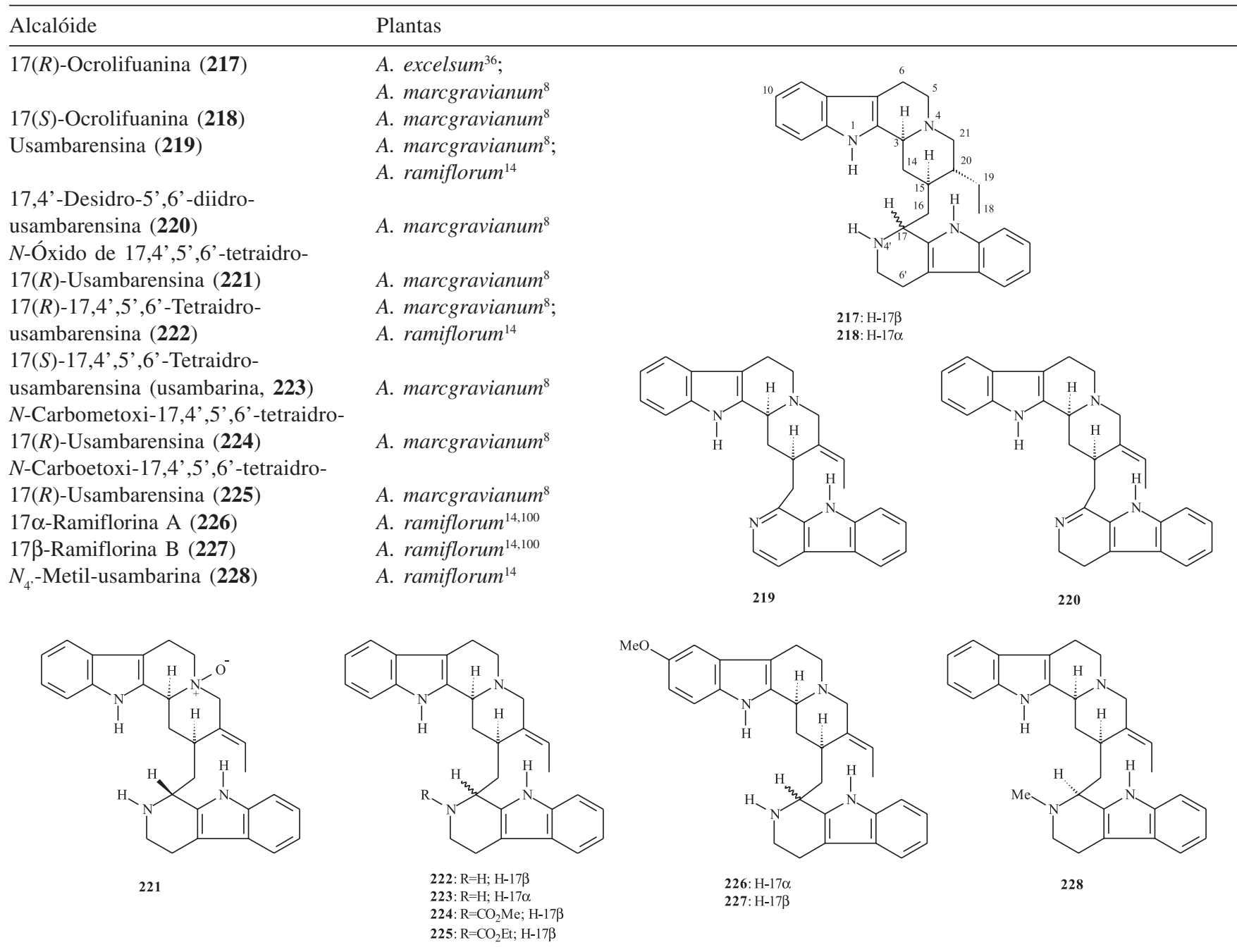

Tabela 18. Alcalóides indólicos dos tipos uleína (229 a 238), aparicina (239 e 240) e valesamina (241)

\begin{tabular}{|c|c|c|}
\hline Alcalóide & Plantas & \\
\hline (+)-Uleína (229) & 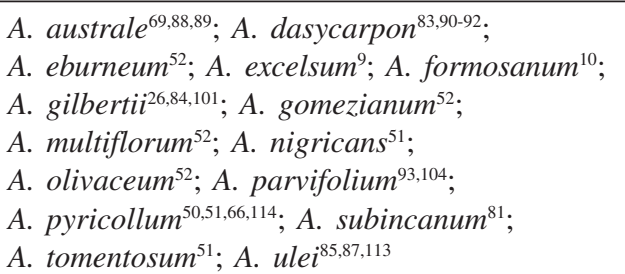 & $\begin{array}{l}\text { I } \\
\text { H }\end{array}$ \\
\hline 3-epi-Uleína (230) & 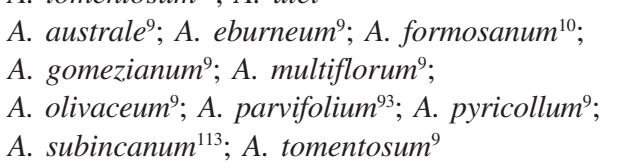 & $\begin{array}{l}\text { 229: } \mathrm{R}^{1}=\mathrm{Et} ; \mathrm{R}^{2}=\mathrm{H} ; \mathrm{R}^{3}=\mathrm{CH}_{2} ; \mathrm{R}^{4}=\mathrm{Me} \\
\text { 230: } \mathrm{R}^{1}=\mathrm{H} ; \mathrm{R}^{2}=\mathrm{Et} ; \mathrm{R}^{3}=\mathrm{CH}_{2} ; \mathrm{R}^{4}=\mathrm{Me} \\
\text { 231: } \mathrm{R}^{1}=\mathrm{Et} ; \mathrm{R}^{2}=\mathrm{R}^{4}=\mathrm{H} ; \mathrm{R}^{3}=\mathrm{CH}_{2} \\
\text { 232: } \mathrm{R}^{1}=\mathrm{Et} ; \mathrm{R}^{2}=\mathrm{H} ; \mathrm{R}^{3}=\mathrm{O} ; \mathrm{R}^{4}=\mathrm{Me} \\
\text { 233: } \mathrm{R}^{1}=\mathrm{Et} ; \mathrm{R}^{2}=\mathrm{H} ; \mathrm{R}^{3}=\mathrm{He} \mathrm{OH} ; \mathrm{R}^{4}=\mathrm{Me}\end{array}$ \\
\hline Des- $N$-metiluleína (231) & $\begin{array}{l}\text { A. dasycarpon } \\
\text { A. } \text { subincanum }^{85,115}\end{array}$ & 234: $\mathrm{R}^{1}=\mathrm{Et} ; \mathrm{R}^{2}=\mathrm{H} ; \mathrm{R}^{3}=\mathrm{He} \mathrm{CH} \mathrm{CH}_{2} \mathrm{OH} ; \mathrm{R}^{4}=\mathrm{Me}$ \\
\hline Dasicarpidona (232) & 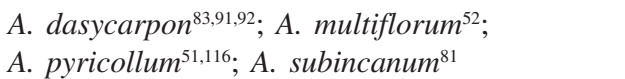 & \\
\hline \multicolumn{3}{|c|}{ Diidrodasicarpidona (dasicarpidol; 233) A. dasycarpon ${ }^{83,91,92}$} \\
\hline Des- $N$-metildasicarpidona (234) & A. dasycarpon ${ }^{83,91,92}$ & \\
\hline Diidrouleína $(\mathbf{2 3 5})$ & A. dasycarpon $^{108} ;$ A. $_{\text {gilbertii }}{ }^{101} ;$ A. nigricans $^{51}$ & $p_{1}^{1}$ \\
\hline 1,13-Diidro-13-hidroxiuleína (236) & $\begin{array}{l}\text { A. dasycarpon } \\
\text { A. } \text { nigricans }^{83,91,92} ; \text { A. formosanum } \\
\end{array}$ & $\begin{array}{l}\text { 235: } \mathrm{R}^{1}=\mathrm{R}^{2}=\mathrm{H} \\
\text { 236: } \mathrm{R}^{1}=\mathrm{CH}_{2} \mathrm{OH} ; \mathrm{R}^{2}=\mathrm{Me}\end{array}$ \\
\hline$N$-Metil-diidrouleína (237) & A. parvifolium ${ }^{108}$ & 237: $\mathrm{R}^{1}=\mathrm{H} ; \mathrm{R}^{2}=\mathrm{Me}$ \\
\hline
\end{tabular}


Tabela 18. continuação

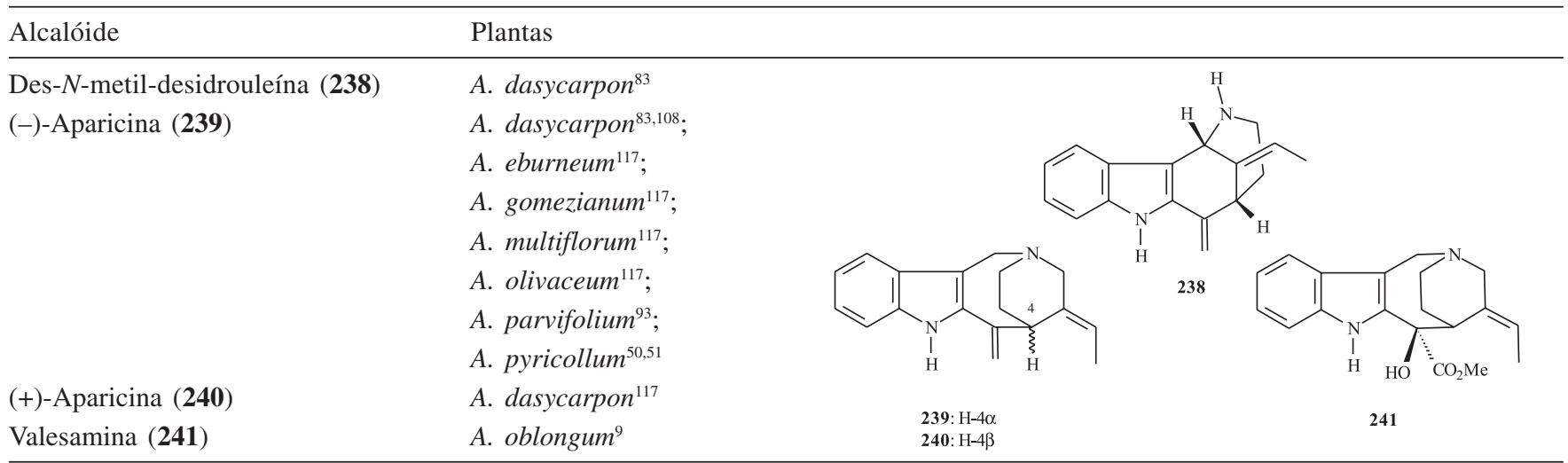

Tabela 19. Alcalóides indólicos de esqueletos não classificados (242 a 247)

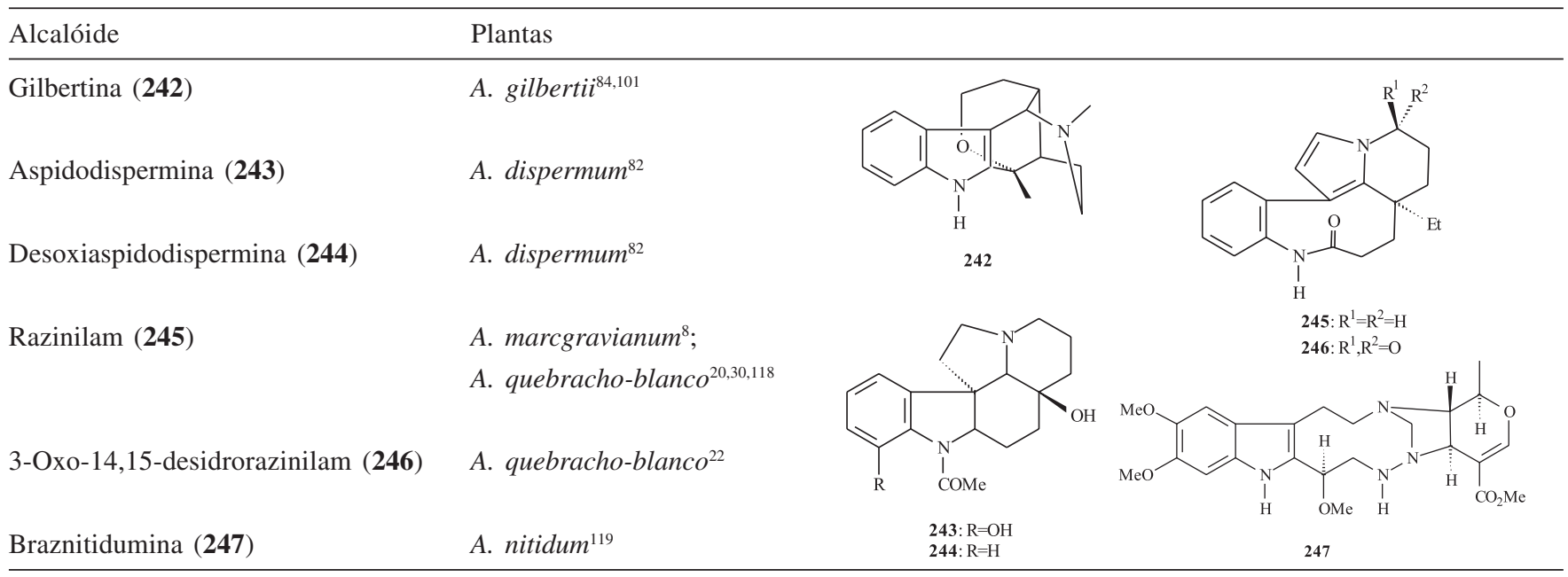

A Tabela 20 apresenta o levantamento dos alcalóides indólicos isolados por espécie e por série de Aspidosperma. Pelos resultados obtidos pôde-se constatar que as espécies do gênero Aspidosperma são caracterizadas pela incidência de uma grande quantidade e diversidade estrutural de alcalóides indólicos. Por essa tabela não se verifica uma distribuição bem caracterizada dos tipos de alcalóides pelas séries taxonômicas, não permitindo, portanto, a classificação das espécies de Aspidosperma em série ser baseada na estrutura química desses alcalóides. Porém, podem ser ressaltados alguns esqueletos básicos em determinadas séries. Na série Nítida observa-se a predominância dos esqueletos aspidospermina, ioimbano e geissoschizol. Na série Polyneura, são encontrados esqueletos do tipo aspidospermina e pirifolidina. Na série Pyricolla, os esqueletos pirifolidina, elipticina-olivacina, uleína e aparicina são encontrados predominantemente. Finalmente, estruturas com o esqueleto aspidoalbina são encontradas na série Nobile.

Tabela 20. Alcalóides indólicos isolados por espécie de Aspidosperma

\begin{tabular}{|c|c|c|c|}
\hline Série/Espécie & Alcalóides & Série/Espécie & Alcalóides \\
\hline$\underline{\text { Rígida }}$ & & A. marcgravianum & $13 ; 15 ; 20 ; 36 ; 73 ; 75 ; 83 ; 96 ; 119 ; 120$ \\
\hline A. rigidum & $122 ; 153 ; 154 ; 155$ & & $121 ; 122 ; 124 ; 125 ; 126 ; 128 ; 129 ; 130$ \\
\hline$\underline{\text { Nítida }}$ & & & $131 ; 132 ; 133 ; 134 ; 135 ; 136 ; 138 ; 139$ \\
\hline$\overline{\text { A. auriculatum }}$ & $121 ; 209$ & & $140 ; 141 ; 142 ; 143 ; 144 ; 164 ; 165 ; 166 ;$ \\
\hline A. carapanauba & 153 & & $167 ; 169 ; 170 ; 189 ; 190 ; 191 ; 192 ; 193 ;$ \\
\hline A. discolor & $\begin{array}{l}11 ; 13 ; 15 ; 17 ; 29 ; 75 ; 120 ; 121 ; 122 \\
128 ; 138 ; 139 ; 151 ; 193 ; 195 ; 196 ; 209 \\
210 ; 212\end{array}$ & & $\begin{array}{l}197 ; 198 ; 199 ; 200 ; 201 ; 202 ; 204 ; 205 \\
209 ; 210 ; 211 ; 212 ; 213 ; 216 ; 217 ; 218 \\
219 ; 220 ; 22 ; 222 ; 223 ; 224 ; 225 ; 245\end{array}$ \\
\hline A. eburneum & $\begin{array}{l}11 ; 15 ; 105 ; 128 ; 138 ; 139 ; 229 ; 230 \\
239\end{array}$ & $\begin{array}{l}\text { A. nitidum } \\
\text { A. oblongum }\end{array}$ & $\begin{array}{l}178 ; 210 ; 247 \\
20 ; 94 ; 120 ; 127 ; 128 ; 137 ; 138 ; 139\end{array}$ \\
\hline A. excelsum & $\begin{array}{l}11 ; 15 ; 85 ; 86 ; 87 ; 119 ; 123 ; 128 ; 136 \\
139 ; 140 ; 168 ; 169 ; 170 ; 171 ; 172 ; 193 \\
217 ; 229\end{array}$ & A. spegazzinii & $\begin{array}{l}145 ; 150 ; 152 ; 189 ; 190 ; 193 ; 198 ; 202 \\
203 ; 204 ; 214 ; 215 ; 241 \\
123 ; 158 ; 160 ; 161\end{array}$ \\
\hline
\end{tabular}

(*) Espécies não incluídas em nenhuma das séries taxonômicas de classificação de Aspidosperma proposta em $1987^{9}$. 
Tabela 20. continuação

\begin{tabular}{ll}
\hline Série/Espécie & Alcalóides \\
\hline Quebrachines & \\
A. chakensis & $3 ; 4 ; 102$ \\
A. quebracho-blanco & $5 ; 7 ; 8 ; 9 ; 10 ; 12 ; 14 ; 24 ; 25 ; 27 ; 30 ; 74$ \\
& $79 ; 84 ; 88 ; 89 ; 90 ; 91 ; 94 ; 95 ; 99 ; 100$ \\
& $101 ; 128 ; 138 ; 139 ; 146 ; 147 ; 148 ; 149$ \\
& $158 ; 163 ; 245 ; 246$
\end{tabular}

Polyneura

A. cuspa

A. cylindrocarpon

A. dispermum

A. peroba

A. polyneuron

A. sessiliflorum

Pyricolla

A. australe

A. gomezianum

A. multiflorum

A. nigricans

A. olivaceum

A. parvifolium

A. populifolium

A. pyricollum

A. pyrifolium

A. quirandy

A. refractum

A. rhombeosignatum

A. subincanum

A. tomentosum

A. ulei

\section{$22 ; 52 ; 53 ; 55 ; 155 ; 164 ; 198$}

$7 ; 9 ; 38 ; 39 ; 40 ; 41 ; 42 ; 43 ; 44 ; 45 ; 46$; $47 ; 48 ; 49 ; 50 ; 51 ; 61$

$243 ; 244$

$8 ; 9 ; 101 ; 128$

$8 ; 9 ; 10 ; 18 ; 101 ; 128 ; 156 ; 157 ; 178$; $179 ; 180 ; 181 ; 182$

8; 9

$8 ; 9 ; 105 ; 111 ; 112 ; 229 ; 230$

15; 229; 230; 239

230; 232; 239

105; 108; 111; 229; 235; 236

20; 105; 111; 229; 230; 239

$110 ; 229 ; 230 ; 231 ; 237 ; 239$

$58 ; 59 ; 60 ; 63 ; 66 ; 67 ; 68 ; 69 ; 88 ; 192$

$8 ; 11 ; 128 ; 138 ; 143 ; 144 ; 162 ; 229 ; 230$; $232 ; 239$

$1 ; 7 ; 8 ; 9 ; 10 ; 13 ; 14 ; 16 ; 18 ; 19 ; 28 ; 29$; $58 ; 61 ; 62 ; 64 ; 65 ; 76 ; 77 ; 84 ; 86 ; 101$; 102

\section{8}

$7 ; 57 ; 58 ; 63 ; 64$

$8 ; 14 ; 17 ; 35 ; 79 ; 82$

92; 104; 105; 109; 110; 114; 115; 116;

$117 ; 187 ; 229 ; 230 ; 231 ; 232$

92; 229; 230

104; 105; 110; 111; 114; 229

\begin{tabular}{ll}
\hline Série/Espécie & Alcalóides \\
\hline $\begin{array}{l}\text { A. vargasii } \\
\text { Nobile }\end{array}$ & $\mathbf{1 0 7} ; \mathbf{1 1 0} ; \mathbf{1 1 1}$ \\
\hline A. &
\end{tabular}

A. album

$8 ; 20 ; 22 ; 34 ; 36 ; 70 ; 71 ; 72 ; 73 ; 74 ; 79$; $80 ; 86 ; 101 ; 102 ; 197 ; 198$

A. desmanthum $\quad \mathbf{7 0} ; \mathbf{7 2} ; \mathbf{7 4}$

A. exalatum

$8 ; 17 ; 19 ; 80 ; 81 ; 82 ; 178 ; 180 ; 181$; 182

A. fendleri $2 ; \mathbf{7 8} ; \mathbf{7 9}$

A. limae

$17 ; 19 ; 20 ; 26 ; 32 ; 33 ; 36 ; 37 ; 73 ; 86$;

92; 96; $97 ; 98$

A. megalocarpon $\quad 20 ; \mathbf{2 1} ; \mathbf{7 3} ; \mathbf{7 9}$

A. melanocalyx $22 ; \mathbf{2 3}$

A. neblinae

$5 ; 6 ; 7 ; 9 ; 10 ; 11 ; 13 ; 14 ; 15 ; 20 ; 24 ; 25$; $31 ; 147 ; 149 ; 177$

A. obscurinervium $\quad 20 ; 26 ; 173 ; 174 ; 175 ; 176$

A. sandwithianum $\mathbf{1 0 1}$

A. spruceanum $\quad \mathbf{7 0} ; \mathbf{7 2} ; \mathbf{7 4}$

Macrocarpa

A. duckei

A. macrocarpon

A. verbascifolium

Outras*

A. campus-belus

A. compactinervium

A. dasycarpon

$52 ; 53 ; 55 ; 56$

$1 ; 52 ; 53 ; 55 ; 67 ; 70$

54

105

85; 93

104; 105; 106; 110; 111; 114; 117; 118 ; $158 ; 159 ; 164 ; 229 ; 231 ; 232 ; 233 ; 234$; 235; 236; 238; 239; 240

A. formosanum

A. gilbertii

A. longepetiolatum

A. pruinosum

20; 229; 230; 236

$104 ; 105 ; 113 ; 188 ; 229 ; 235 ; 242$

105; 111

85; 128; 134; 137; 138; 139; 156; 182; $183 ; 184 ; 185 ; 186 ; 193 ; 194 ; 202 ; 203$; $205 ; 206 ; 207 ; 208 ; 209 ; 210$

A. ramiflorum
$138 ; 139 ; 193 ; 219 ; 222 ; 226 ; 227$; 228

(*) Espécies não incluídas em nenhuma das séries taxonômicas de classificação de Aspidosperma proposta em 1987 .

\section{AGRADECIMENTOS}

Ao Conselho Nacional de Desenvolvimento Científico e Tecnológico (CNPq), à Fundação Coordenação de Aperfeiçoamento de Pessoal de Nível Superior (CAPES), à Fundação de Amparo à Pesquisa do Estado de Minas Gerais (FAPEMIG) e à Fundação de Amparo à Pesquisa do Estado do Amazonas (FAPEAM).

\section{REFERÊNCIAS}

1. Rapini, A.; Tese de Doutorado, Universidade de São Paulo, Brasil, 2000.

2. Nicholas, A.; Baijnath, A.; Botanical Rev. 1994, 60, 440.

3. Rocha, A. I.; Reis-Luz, A. I.; Rodrigues, W. A.; Acta Amaz. 1982, 12, 381.

4. Struwe, L.; Albert, V. A.; Bremer, B.; Cladistics 1994, 10, 175.

5. Ribeiro, J. E. L. S.; Hopkins, M. J. G.; Vicentini, A.; Sothers, C. A.; Costa, M. A. S.; Brito, J. M.; Souza, M. A. D.; Martins, L. H. P.; Lohmann, L. G.; Assunção, P. A. C. L.; Pereira, E. C.; Silva, C. F.; Mesquita, M. R.; Procópio, L. C.; Flora da Reserva Ducke: Guia de Identificação das Plantas Vasculares de uma Floresta de Terra-Firme na Amazônia Central, INPA: Manaus, 1999.

6. Campbell, D. G.; Hammond, H. D.; Floristic Inventory of Tropical Countries: The Status of Plant systematics, Collections, and Vegetation, plus Recommendations for the Future, NY Botanical Garden: New York, 1989.

7. Corrêa, M. P.; Dicionário das Plantas Úteis do Brasil, Imprensa Nacional: Rio de Janeiro, 1926.
8. Robert, G. M. T.; Ahond, A.; Poupat, C.; Potier, P.; Jousselin, A.; Jacquemin, H.; J. Nat. Prod. 1983, 46, 694.

9. Bolzani, V. S.; Serur, L. M.; Matos, F. J. A.; Gottlieb, O. R.; Biochem. Syst. Ecol. 1987, 15, 187.

10. Garcia, M.; Ruben, F.; Brown, K. S.; Phytochemistry 1976, 15, 1093.

11. Albuquerque, B. W. P.; Acta Amaz. 1971, 1, 9.

12. Gottlieb, O. R.; Biochem. Syst. Ecol. 1978, 6, 185.

13. Weniger, B.; Robledo, S.; Arango, G. J.; Deharo, E.; Aragon, R.; Muñoz, V.; Callapa, J.; Lobstein, A.; Anton, R.; J. Ethnopharmacol. 2001, 78, 193.

14. Ferreira, I. C. P.; Lonardoni, M. V. C.; Machado, G. M. C.; Leon, L. L.; Gobbi-Filho, L.; Pinto, L. H. B.; Oliveira, A. J. B.; Mem. Inst. Oswaldo Cruz 2004, 99, 325.

15. Oliveira, F. Q.; Junqueira, R. G.; Stehmann, J. R.; Brandão, M. G. L.; Rev. Bras. Plant. Med. 2003, 5, 23.

16. Bourdy, G.; Oporto, P.; Gimenez, A.; Deharo, E.; J. Ethnopharmacol. 2004, 93, 269.

17. Brandão, M. G. L.; Grandi, T. S. M.; Rocha, E. M. M.; Sawyer, D. R.; Krettli, A. U.; J. Ethnopharmacol. 1992, 36, 175.

18. Carvalho, L. H.; Brandão, M. G. L.; Santos-Filho, D.; Lopes, J. L. C.; Krettli, A. U.; Braz. J. Med. Biol. Res. 1991, 24, 1113.

19. Deutsch, H. F.; Evenson, M. A.; Drescher, P.; Sparwasser, C.; Madsen, P. O.; J. Pharm. Biomed. Anal. 1994, 12, 1283.

20. Lyon, R. L.; Fong, H. H. S.; Farnsworth, N. R.; Svoboda, G. H.; J. Pharm. Sci. 1973, 62, 218.

21. Lyon, R. L.; J. Pharm. Sci. 1973, 62, 833.

22. Aimi, N.; Uchida, N.; Ohya, N.; Hosokawa, H.; Takayama, H.; Sakai, S.; Tetrahedron Lett. 1991, 37, 4949.

23. Allen, J. R. F.; Holmstedt, B. R.; Phytochemistry 1980, 19, 1573. 
24. Wenkert, E.; J. Am. Chem. Soc. 1962, 84, 98.

25. Nunes, D. S.; Koike, L.; Taveira, J. J.; Reis, F. A. M.; Phytochemistry 1992 31,2507

26. Duarte, A. P.; Miranda, E. C.; An. Acad. Brasil. Ciênc. 1983, 55, 189.

27. Mitaine, A. C.; Mesbah, K.; Richard, B.; Petermann, C.; Arrazola, S.; Moretti, C.; Hanrot, M. Z.; Oliver, L. L. M.; Planta Med. 1996, 62, 458.

28. Rivas, P.; Cassels, B. K.; Morello, A.; Repetto, Y.; Biochem. Phys., Part C 1999, 122, 27.

29. Biel, J. H.; Drukker, A. E.; Mitchell, T. F.; Sprengeler, E. P.; Nuher, P. A.; Conway, A. C.; Horita, A.; J. Am. Chem. Soc. 1959, 81, 2805.

30. Benoit, P. S.; Angry, G.; Lyon, R. L.; Fong, H. H. S.; Farnsworth, N. R.; J. Pharmacol. Sci.. 1973, 62, 1889.

31. Garrett, R. H.; Grisham, C. M.; Biochemistry, Saunders Coll. Publishing: Orlando, 1995.

32. Foye, W. O.; Lemke, T. L.; Williams, D. A.; Medicinal Chemistry, Williams \& Wilkins: Media, 1995.

33. Craveiro, A. A.; Matos, F. J. A.; Serur, L. M.; Phytochemistry 1983, 22, 1526.

34. Picada, J. N.; Silva, K. V. C. L.; Erdtmann, B.; Henriques, A. T.; Henriques, J. A. P.; Mutat. Res. 1997, 379, 135.

35. Verpoorte, R.; Ruigrok, C. L. M.; Svendsen, A. B.; Planta Med. 1982, 46, 149

36. Verpoorte, R.; Kos-Kuyck, E.; Tsoi, A. T. A.; Ruigrok, C. L. M.; Jong, G.; Svendsen, A. B.; Planta Med. 1983, 48, 283.

37. Gilbert, B.; An. Acad. Brasil. Ciênc. 1966, 38, 315.

38. Manske, R. H. F.; Rodrigo, R.; The Alkaloids, Academic Press: New York, 1965.

39. Jácome, R. L. R. P.; Tese de Doutorado, Universidade Federal de Minas Gerais, Brasil, 1998.

40. http://amazonas.rds.org, acessada em Junho de 2005.

41. Djerassi, C.; Brewer, H. W.; Budzikiewicz, H.; Orazi, O. O.; Corral, R. A.; J. Am. Chem. Soc. 1962, 84, 3480.

42. Schnoes, H. K.; Burlingame, A. L.; Biemann, K.; Tetrahedron Lett. 1962, 993.

43. Thomas, D. W.; Schnoes, J. K.; Biemann, K.; Experientia 1969, 25, 678.

44. Djerassi, C.; Archer, A. A. P. G.; George, T.; Gilbert, B.; Antonaccio, L. D.; Tetrahedron 1961, 16, 212.

45. Antonaccio, L. D.; Pereira, N. A.; Gilbert, B.; Vorbrueggen, H.; Budzikiewicz, H.; Wilson, J. M.; Durham, L. J.; Djerassi, C.; J. Am. Chem. Soc. 1962, 84, 2161.

46. Biemann, K.; Spiteller-Friedmann, M.; Spiteller, G.; Tetrahedron Lett. 1961, 485.

47. Biemann, K.; Spiteller-Friedmann, M.; Spiteller, G.; J. Am. Chem. Soc. 1963, 85, 631 .

48. Dastoor, N. J.; Gorman, A. A.; Schmid, H.; Helv. Chim. Acta 1967, 50, 213.

49. Ferreira, J. M.; Gilbert, B.; Owellen, R. J.; Djerassi, C.; Experientia 1963, 19, 585.

50. Arndt, R. R.; Djerassi, C.; Experientia 1965, 21, 566.

51. Arndt, R. R.; Brown, S. H.; Ling, N. C.; Roller, P.; Djerassi, C.; FerreiraFilho, J. M.; Gilbert, B.; Miranda, E. C.; Flores, S. E.; Duarte, A. P.; Carrazzoni, E. P.; Phytochemistry 1967, 6, 1653.

52. Gilbert, B.; Duarte, A. P.; Nakagawa, Y.; Joule, J. A.; Flores, S. E.; Brissolese, J. A.; Campello, J.; Carrazzoni, E. P.; Owellen, R. J.; Blossey, E. C.; Brown, Jr., K. S.; Djerassi, C.; Tetrahedron 1965, 21, 1141.

53. Walser, A.; Djerassi, C.; Helv. Chim. Acta 1965, 48, 391.

54. Di Genova, L.; Planta Med. 1979, 37, 165.

55. Pinar, M.; Bycroft, B. W.; Seibl, J.; Schmid, H.; Helv. Chim. Acta 1965, $48,822$.

56. McLean, S.; Palmer, K.; Marion, L.; Can. J. Chem. 1960, 38, 1547.

57. Brown, K. S.; Djerassi, C.; J. Am. Chem. Soc. 1964, 86, 2451.

58. Burnell, R. H.; Medina, J.; Phytochemistry 1968, 7, 2045.

59. Miranda, E. C.; Gilbert, B.; Experientia 1969, 25, 575.

60. Antonaccio, L. D.; Budzikiewicz, H.; Chem. Ber. 1962, 93, 962.

61. Taylor, W. I.; Raab, N.; Lehner, H.; Schmutz, J.; Helv. Chim. Acta 1959, $42,2750$.

62. Cava, M. P.; Nomura, K.; Talapatra, S. K.; Tetrahedron 1964, 20, 581.

63. Pinar, M.; von Philipsborn, W.; Vetter, W.; Schmid, H.; Helv. Chim. Acta 1962, 45, 2260

64. Djerassi, C.; Antonaccio, L. D.; Budzikiewicz, H.; Wilson, J. M.; Gilbert, B.; Tetrahedron Lett. 1962, 1001.

65. Djerassi, C.; Owellen, R. J.; Ferreira, J. M.; Antonaccio, L. D.; Experientia 1962, 18, 397.

66. Gilbert, B.; Antonaccio, L. D.; Archer, A. A. P. G.; Djerassi, C.; Experientia 1960, 16, 61 .

67. Gilbert, B.; Ferreira, J. M.; Owellen, R. J.; Swanholm, C. E.; Budzikiewicz, H.; Durham, L. J.; Djerassi, C.; Tetrahedron Lett. 1962, 59.

68. Djerassi, C.; Gilbert, B.; Shoolery, J. N.; Johnson, L. F.; Biemann, K.; Experientia 1961, 17, 162.
69. Ondetti, M. A.; Deulofeu, V.; Tetrahedron Lett. 1961, 160.

70. Gilbert, B.; Antonaccio, L. D.; Djerassi, C.; J. Org. Chem. 1962, 27, 4702.

71. Mills, J. F. D.; J. Chem. Soc. 1960, 1458

72. Simões, J. C.; Gilbert, B.; Cretney, W. J.; Hearn, M.; Kutney, J. P.; Phytochemistry 1976, 15, 543

73. Djerassi, C.; George, T.; Finch, N.; Lodish, H. F.; Budzikiewicz, H.; Gilbert, B.; J. Am. Chem. Soc. 1962, 84, 1499.

74. Antonaccio, L. D.; J. Org. Chem. 1960, 25, 1262.

75. Mitaine, A.-C.; Weninger, B.; Sauvain, M.; Lucumi, E.; Aragon, R.; ZechesHanrot, M.; Planta Med. 1998, 64, 487.

76. Mitaine, A. C.; Weniger, B.; Sauvain, M.; Lucumi, E.; Aragón, R.; ZechesHanrot, M. Z.; Planta Med. 1998, 64, 487.

77. Sanchez, L.; Wolfango, E.; Brown, K. S.; An. Acad. Brasil. Ciênc. 1971, 43,603 .

78. Brown, K. S.; Sanches, L. W. E.; Figueiredo, A. A.; Ferreira-Filho, J. M.; J. Am. Chem. Soc. 1966, 88, 4984.

79. Pinar, P.; Chem. Soc. Rev. 1992, 113

80. Pinar, M.; Schmid, H.; Helv. Chim. Acta 1967, 50, 89.

81. Gaskell, A. J.; Joule, J.; Tetrahedron Lett. 1970, 1, 77.

82. Ikeda, M.; Djerassi, C.; Tetrahedron Lett. 1968, 5837.

83. Joule, J. A.; Ohashi, M.; Gilbert, B.; Djerassi, C.; Tetrahedron 1965, 21, 1717.

84. Mukhopadhyay, S.; Cordell, G. A.; Tetrahedron 1983, 39, 3639

85. Büchi, G.; Warnhoff, E. W.; J. Am. Chem. Soc. 1959, 81, 4433.

86. Büchi, G.; Mayo, D. W.; Hochstein, F. A.; Tetrahedron 1961, 15, 167.

87. Woodward, R. B.; Iacobucci, G. A.; Hochstein, F. A.; J. Am. Chem. Soc. 1959, $81,4434$.

88. Ondetti, M. A.; Deulofeu, V.; Tetrahedron Lett. 1959, 1.

89. Ondetti, M. A.; Deulofeu, V.; Tetrahedron Lett. 1960, 18

90. Ohashi, M.; Toshikazu, O.; Expert Opin. Ther. Pat. 1996, 6, 1285.

91. Ohashi, M.; Joule, J. A.; Gilbert, B.; Djerassi, C.; Experientia 1964, 20, 363.

92. Bick, I. R. C.; Experientia 1964, 20, 363.

93. Jácome, R. L. R. P.; Oliveira, A. B.; Raslan, D. S.; Wagner, H.; Quim. Nova 2004, 27, 897.

94. Ferreira, P. C.; Marini-Bettolo, G. B.; Schmutz, J.; Experientia 1959, 15, 179.

95. Dastoor, N.; Schmid, H.; Experientia 1963, 19, 297.

96. Gilbert, B.; Brissolese, J. A.; Finch, N.; Taylor, W. I.; Budzikiewicz, H.; Wilson, J. M.; Djerassi, C.; J. Am. Chem. Soc. 1963, 85, 1523.

97. Olivier, L.; Lévy, J.; Le Men, J.; Janot, M. M.; Budzikiewicz, H.; Djerassi, C.; Bull. Soc. Chim. France 1965, 868.

98. Benoin, P. R.; Burnell, R. H.; Medina, J. D.; Can. J. Chem. 1967, 45, 725 99. Burnell, R. H.; Sen, N-T.; Phytochemistry 1971, 10, 895.

100. Fátima, M.; Marques, S.; Kato, L.; Leitão-Filho, H. F.; Reis, F. A. M.; Phytochemistry 1996, 41, 963.

101. Miranda, E. C.; Blechert, S.; Tetrahedron Lett. 1982, 23, 5395.

102. Britten, A. Z.; Smith, G. F.; Spiteller, G.; Chem. Ind. 1963, 1492.

103. Olivier, L.; Lévy, J.; Le Men, J.; Janot, M. M.; Ann. Pharm. Franç. 1962, 20,361 .

104. Joule, J. A.; Djerassi, C.; J. Chem. Soc. 1964, 2777.

105. Markey, S.; Biemann, K.; Witkop, B.; Tetrahedron Lett. 1967, 157.

106. Orazi, O. O.; Corral, R. A.; Stoichevich, M. E.; Can. J. Chem. 1966, 44, 1523.

107. Sandoval, A.; Walls, F.; Shoolery, J. N.; Wilson, J. M.; Budzikiewicz, H.; Djerassi, C.; Tetrahedron Lett. 1962, 409.

108. Ohashi, M.; Joule, J. A.; Djerassi, C.; Tetrahedron Lett. 1964, 3899.

109. Kahrl, J.; Gebreyesus, T.; Djerassi, C.; Tetrahedron Lett. 1971, 2527.

110. Biemann, K.; Spiteller, G.; Tetrahedron Lett. 1961, 299.

111. Pereira, M. M.; Tese de Doutorado, Universidade Federal de Minas Gerais, Brasil, 2005

112. Pereira, M. M.; Souza Júnior, S. N.; Alcântara, A. F. C.; Pilo-Veloso, D.; Alves, R. B.; Machado, P. O.; Azevedo, A. O.; Moreira, F. H.; Castro, M. S. A.; Raslan, D. S.; Rev. Bras. Plantas Med., 2006, 8, 1.

113. Büchi, G.; Gould, S. J.; Näf, F.; J. Am. Chem. Soc. 1971, 93, 2492.

114. Schmutz, J.; Hunziker, F.; Hirt, R.; Helv. Chim. Acta 1957, 40, 1189.

115. Gaskell, A. J.; Joule, J. A.; Chem. Ind. 1967, 1089.

116. Goodwin, S.; Smith, A. F.; Horning, E. C.; J. Am. Chem. Soc. 1959, 81, 1903.

117. Joule, J. A.; Monteiro, H.; Durham, L. J.; Gilbert, B.; Djerassi, C.; J. Chem. Soc. $\mathbf{1 9 6 5}, 4773$

118. Abraham, D. J.; Rosenstein, R. D.; Lyon, R. L.; Fong, H. H. S.; Tetrahedron Lett. 1972, 909.

119. Pereira, M. M.; Alcântara, A. F. C.; Piló-Veloso, D.; Raslan, D. S.; J. Braz. Chem. Soc. 2006, 17, 1274. 